ROBUST STABILIZATION OF A UNIFORMLY ROTATING RIGID BODY

\author{
By \\ Rongze Zhao \\ and \\ Thomas A. Posbergh
}

IMA Preprint Series \# 1185

November 1993 


\title{
Robust Stabilization of a Uniformly Rotating Rigid Body
}

\author{
Rongze Zhao* \\ Control Sciences and Dynamical Systems \\ University of Minnesota, Minneapolis, MN 55455 \\ and \\ Thomas A. Posbergh* \\ Department of Aerospace Engineering and Mechanics \\ University of Minnesota, Minneapolis, MN 55455
}

* Research supported in part by NSF/MSS-9009707 and by the University of Minnesota Graduate School Grant-in-Aid Program. 


\begin{abstract}
$\S$ Abstract
This paper discusses the robust stabilization by feedback control of a rigid body in uniform rotation about an arbitrary axis. We consider a linear control law for which the closed loop system is hamiltonian. For such systems, the energy-momentum method enables the selection of feedback gains which ensure stability of uniform rotation about an arbitrary axis. The stability is proved robust with respect to parametric uncertainty in the mass and inertia values. We also show that there exists special cases for which one applied torque is adequate for stabilization. The methodology is illustrated with several examples.
\end{abstract}




\section{$\S 1$. Introduction}

This paper presents an approach to the design of robust control laws for stabilizing the uniform rotation of a rigid body. This research seeks to further the understanding of fundamental issues related to the dynamics and control of spacecraft. Such problems are fundamentally nonlinear. Our approach is based on the energy-momentum method which is eminently suited for exploiting the underlying geometric structures in the context of hamiltonian systems with symmetry. The conditions ensuring stability of the system are naturally exploited to ensure robustness of the stabilizing control law with respect to parameter variations.

The problem of stabilization and stability analysis of rigid bodies, systems of rigid bodies and rigid bodies with attached, flexible appendages has received a great of deal of attention in recent years. Much of this work deals with the stabilization of a single rigid body about zero angular velocity in a body frame aligned with the principal axes of inertia. This approach is taken in, for example, [2], [3], [9], [10] and [16]. In [9], the asymptotic stabilization of rotation about a principal axis was studied. The problem of stabilizing the uniform rotation of a rigid body about the intermediate principal axis of inertia was investigated in [6], [7]. We note that similar problems were investigated for gyroscopic systems in a Lagrangian setting in [26] and [27]. An important generalization of this problem is the stabilization of uniform rotation about an arbitrary axis fixed in the body frame. In [18] and [28], we investigated a family of quadratic feedback control laws which solve this problem. In the present paper, we investigate a family of linear control laws stabilizing a uniform rotation about an arbitrary axis, and we prove the robustness with respect to parametric uncertainty. Preliminary results of our work are reported in [29].

Much of existing literature on the control of uniform rigid body rotation assumes exact knowledge of the rigid body inertia and is concerned with stabilizing uniform rigid body rotation about the intermediate axis of inertia. In contrast, in this paper we consider the design of stabilizing feedback control laws in the presence of parametric uncertainty in the rigid body inertia and deal with the stabilization of uniform rotation about an arbitrary axis. In many applications the principal axes of inertia may not coincide with the desired axis of rotation. An example is rotation about the roll axis of the space shuttle (see $\S 5$ below).

The reduction of the number of control torques is of interest for theoretical and practical reasons. Theoretically, if three independent applied torques are available, then the stabilization problem can be solved by the Lyapunov's first method (see $\S 4$ below). Moreover, in the case when 
one or two actuators fail and less than three applied torques are available, we have to use the remaining applied torque(s) to stabilize the rotation. Previous work, such as [2] and [8], reported the results of stabilizing zero angular velocity by one torque. In [6] and [7] the authors used only one torque to stabilize the rotation about the intermediate principal axis. In [25] the authors solved the stabilization of rotation about an arbitrary axes by two torques. All this work assumes a principal-axes coordinate system. However, this coordinate frame may not be convenient in practical applications. In this paper we work with a general coordinate frame and show that if the axis of rotation lies in a coordinate plane, then we can stabilize the rotation by only one applied torque aligned with a coordinate axis.

The method of robust control design employed in this work is based on the energy-momentum methodology as developed in [21]. We design a family of feedback control laws such that the closed loop system remains hamiltonian. The energy-momentum method can then be applied to determine criteria for the stability of the closed loop system. These criteria are in the form of inequalities involving the parameter values of the stabilizing control laws and the inertia values of the rigid body. In a subsequent paper we shall show that the energy-momentum method has application in stabilizing a coupled system, such as a rotating rigid body with an attached flexible appendage.

The paper is organized as follows: In $\S 2.1$ - $\S 2.2$ we introduce preliminary material. In $\S 2.1$, the geometric framework for rigid body dynamics is summarized. In $\S 2.2$ we describe the energymomentum method as it is used in this paper. In $\S 3$, we apply the energy-momentum method to analyze the stability of the closed loop system and find the control laws which stabilize uniform rotation about an arbitrary axis fixed in the body frame. In $\S 4$ we discuss the reduction of the number of control torques. Two numerical examples and simulations are presented in $\S 5$. In $\S 6$ we discuss robust stability: In $\S 6.1$ we introduce the concept of reduced space and relate formal stability and Lyapunov stability in the context of this paper, $\S 6.1$ is a continuation of $\S 2.2$. In $\S 6.2$, we define robustness for a family of control laws, then prove that the family of control laws presented in $\S 3$ is robust in this sense. We conclude with $\S 7$.

\section{$\S 2$. Notation and Background Material}

In the first half of this section, we outline the notation and summarize the results used in our subsequent analysis of rigid body motion. In the second half, we introduce the energy-momentum method. References for this section include [1], [4], [12], [14], [21], [22], [23] and [26]. Our notation is consistent with [1]. 


\section{§2.1. Rotation group $S O(3)$ and its Lie algebra so(3)}

The configuration space of a rigid body in rotation about a fixed point is $S O(3):=\left\{\mathbf{A} \in \mathbb{R}^{3 \times 3}\right.$ : $\left.\mathbf{A A}^{T}=\mathbf{1}_{3}, \operatorname{det}(\mathbf{A})=1\right\}$, the set of all orthogonal matrices with determinant 1 . Each element $\mathbf{A} \in S O(3)$ represents a particular position or configuration of the rigid body. Associated to any element $\mathbf{A} \in S O(3)$, we have the tangent space at the current configuration $T_{\mathbf{A}} S O(3)$. The tangent space to $S O(3)$ at $1_{3}$, the $3 \times 3$ identity matrix, is the Lie algebra of $S O(3)$ and is denoted by $s o(3)$, the set of all skew-symmetric real matrices. As a vector space, so(3) corresponds to the space of angular velocity, which we identify with $\mathbb{R}^{3}$ by the isomorphism $S: \mathbb{R}^{3} \rightarrow s o(3)$ defined by

$$
\mathbf{m} \mapsto S(\mathbf{m})=\left[\begin{array}{ccc}
0 & -m_{3} & m_{2} \\
m_{3} & 0 & -m_{1} \\
-m_{2} & m_{1} & 0
\end{array}\right], \text { where } \mathbf{m}=\left[\begin{array}{c}
m_{1} \\
m_{2} \\
m_{3}
\end{array}\right] \in \mathbb{R}^{3} .
$$

Properties of this isomorphism can be found in [6] and [26]. The dual space to $T_{\mathbf{A}} S O(3)$ is denoted by $T_{\mathbf{A}}^{*} S O(3)$. The dual space to $s o(3)$ is denoted by $s o^{*}(3)$, which corresponds to the angular momentum space. We define the tangent bundle by $T S O(3):=\bigcup_{\mathbf{A} \in S O(3)} T^{*} S O(3)$. The cotangent bundle $T^{*} S O(3)$ is defined as $T^{*} S O(3):=\bigcup_{\mathbf{A} \in S O(3)} T_{\mathbf{A}}^{*} S O(3)$, which is also the phase space of the rigid body.

The following facts will be used in the next section. Their proofs are routine.

Facts. For fixed $\mathbf{A} \in S O(3)$,

$$
\begin{aligned}
& \text { (i) } T_{\mathbf{A}}^{*} S O(3) \cong T_{\mathbf{A}} S O(3) \cong s o^{*}(3) \cong s o(3) \cong \mathbb{R}^{3} \\
& \text { (ii) } T_{\mathbf{A}} S O(3)=s o(3) \mathbf{A}=\mathbf{A} s o(3) \\
& \text { (iii) } T S O(3) \cong S O(3) \times \mathbb{R}^{3} \cong T^{*} S O(3)
\end{aligned}
$$

A motion is a curve in configuration space parametrized by time $t$. For any motion, $\mathbf{A}(t) \in$ $S O(3), t \in[0,+\infty), \dot{\mathbf{A}}(t)=\frac{d \mathbf{A}(t)}{d t} \in T_{\mathbf{A}} S O(3)$. From (ii), $\dot{\mathbf{A}}(t)=\boldsymbol{\xi} \mathbf{A}=\mathbf{A} \boldsymbol{\eta}$, for some $\boldsymbol{\xi}, \boldsymbol{\eta} \in$ so(3). Here $\boldsymbol{\xi}=\dot{\mathbf{A}} \mathbf{A}^{-1}$ and $\boldsymbol{\eta}=\mathbf{A}^{-1} \dot{\mathbf{A}}$ correspond respectively to the spatial and body representations of the angular velocity at time $t$ (cf. [1],[4]). We use lower case letters for spatial quantities, upper case for body quantities consistent with the notation in [13]. Thus, we write $\boldsymbol{\xi}=S(\boldsymbol{\omega}), \boldsymbol{\eta}=S(\Omega)$, where $\boldsymbol{\omega}, \boldsymbol{\Omega} \in \mathbf{R}^{3}$. By the definitions of $\boldsymbol{\xi}$ and $\boldsymbol{\eta}$, we have $\boldsymbol{\xi}=\mathbf{A} \boldsymbol{\eta} \mathbf{A}^{-1}$. By applying the identity $\mathbf{A} S(\boldsymbol{\Omega}) \mathbf{A}^{-1}=S(\mathbf{A} \Omega)$, we get $\boldsymbol{\omega}=\mathbf{A} \Omega$, a relationship between spatial and body angular velocity vectors. The pairing between $T_{\mathbf{A}}^{*} S O(3)$ and $T_{\mathbf{A}} S O(3)$ is defined

$$
\langle S(\boldsymbol{\pi}) \mathbf{A}, S(\boldsymbol{\omega}) \mathbf{A}\rangle:=\frac{1}{2} \operatorname{tr}\left[(S(\boldsymbol{\pi}) \mathbf{A})^{T}(S(\boldsymbol{\omega}) \mathbf{A})\right]=\frac{1}{2} \operatorname{tr}\left[(S(\boldsymbol{\pi}))^{T} S(\boldsymbol{\omega})\right]=\boldsymbol{\pi} \cdot \boldsymbol{\omega}
$$


where, $S(\boldsymbol{\pi}) \mathbf{A} \in T_{\mathbf{A}}^{*} S O(3), S(\boldsymbol{\omega}) \mathbf{A} \in T_{\mathbf{A}} S O(3)$ and $\operatorname{tr}[\cdot]$ denotes the trace of the matrix argument. For $\mathbf{A}=\mathbf{1}_{3}$, this defines the duality pairing between $s o^{*}(3)$ and $s o(3)$. From (i) and (ii), we have $T_{\mathbf{A}}^{*} S O(3) \cong s o(3) \mathbf{A}=\mathbf{A} s o(3)$. Thus, we identify an element in $T_{\mathbf{A}}^{*} S O(3)$ with $S(\boldsymbol{\pi}) \mathbf{A}$ or $\mathbf{A} S(\Pi)$ corresponding to spatial or body representation, respectively.

\section{§2.2. The energy-momentum method}

Here we briefly describe the energy-momentum method. For more details refer to [1], [4], [5] and [21]. Familiarity with the modern geometric theory of hamiltonian systems found in [1] is assumed. For the reference of those not familiar with this theory, we have included a summary of basic results used in this paper in Appendix 1.

The classical work of Lagrange, Dirichlet, et al. deals with equilibrium and stability theory by an energy method, which finds equilibrium points and assesses their stability by finding the critical points and extremals of an energy functional. However, the stability analysis of a rotating rigid body shows that in addition to the energy, another conserved quantity-the angular momentumshould be taken in to account as well (cf.[4], [19]). The generalization of the angular momentum is the concept of momentum map. This concept is related to the action of a Lie group (a group which is also a manifold and has smooth multiplication and inverse operations). In our context, the Lie group will be the rotation group $S O(3)$ and its isotropy subgroup $S_{\mu}(3)$ (to be defined below). A group action is a rotation from one configuration to another one. This idea is formalized in the following definition:

Definition 2.1. Let $Q$ be a smooth manifold. A (rotational) $S O(3)$-action on $Q$ is a smooth mapping $\Phi: S O(3) \times Q \rightarrow Q$ such that:

(i) for all $x \in Q, \Phi(e, x)=x$, where $e$ is the identity and $x \in Q$,

(ii) for every $A, B \in S O(3), \Phi(A,(B, x))=\Phi(A B, x)$ for all $x \in Q$.

In the context of this paper, the configuration space $Q$ happens to be $S O(3)$. Thus, the rotational $S O(3)$-action $\Phi$ on $Q$ is left matrix multiplication.

If there is no possibility of confusion, we shall denote $\Phi(A, x)$ by $\mathbf{A} \cdot \mathbf{x}$. Thus, an element $\mathbf{A} \in$ $S O(3)$ acting on $\mathrm{x} \in Q$ rotates the current configuration represented by $\mathrm{x}$ to a new configuration represented by $\mathbf{A} \cdot \mathbf{x}$. We are interested in the set consisting of all points which can be reached from $\mathrm{x}$ under an $S O(3)$-action. This set will be called the $S O(3)$ - orbit. Sometimes we are only interested in the subset of an $S O(3)$-orbit which consists of those points that can be reached under 
the action applied by a subgroup $G$ of $S O(3)$. This subset of $S O(3)$ will be called the $G$ - orbit. Formally we introduce the following.

Definition 2.2. Let $G$ be a subgroup of $S O(3)$ and act on $Q$. For $x \in Q$, the $G$-orbit of $x$ is given by

$$
[x]_{G}:=\{A \cdot x: A \in G\}
$$

We also denote the $G$-orbit of $x$ by $G \cdot x$. If $G=S O(3)$, then the $G$-orbit is called $S O(3)$-orbit.

The relation of belonging to the same $G$-orbit is an equivalence relation (cf. [1]). The set of equivalence classes (i.e., the set of orbits) is denoted by $P / G$.

Consider an $S O(3)$-action $\Phi$ on $Q$. Corresponding to $\boldsymbol{\xi} \in s o(3)$, the exponential matrix is $\exp (\boldsymbol{\xi}):=e^{\xi} \in S O(3)$ (see [1]). The $S O(3)$-action then induces an infinitesimal rotation with angular velocity corresponding to $\boldsymbol{\xi} \in S O(3)$ in the following way:

Definition 2.3. Let $\Phi$ be the $S O(3)$-action on $Q, \boldsymbol{\xi} \in \operatorname{so}(3)$ is fixed. For any $\mathbf{x} \in Q, \boldsymbol{\xi}_{Q}(\mathbf{x}):=$ $\left.\frac{d}{d t}(\exp (t \boldsymbol{\xi}) \cdot \mathbf{x})\right|_{t=0}=\left.\frac{d}{d t} \Phi(\exp (t \boldsymbol{\xi}), \mathbf{x})\right|_{t=o}$ is called the infinitesimal generator of the $S O(3)$-action $\Phi$ corresponding to angular velocity $\boldsymbol{\xi} \in$ so(3).

Note that $\boldsymbol{\xi}_{Q}$ is a vector field on $Q$. Now we are ready to give the definition of momentum map.

Definition 2.4. Let $\Phi$ be the $S O(3)$-action on the smooth manifold $Q$. Let $P=T^{*} Q$. The map $\alpha: P \rightarrow s o(3)^{*}$ is called a momentum map relative to $S O(3)$, if

$$
\langle\boldsymbol{\alpha}(q, p), \boldsymbol{\xi}\rangle=\left\langle p, \boldsymbol{\xi}_{Q}\right\rangle
$$

for every $\boldsymbol{\xi} \in$ so(3), $z=(q, p) \in P=T^{*} Q$, where $\boldsymbol{\xi}_{Q} \in T Q$ is the infinitesimal generator of the $S O(3)$-action $\Phi$ on $Q$ corresponding to $\xi \in s o(3),\langle\cdot, \cdot\rangle$ is the duality pairing defined by (2.1).

Remark. This definition is similar to the definition of the momentum map of vakonomic mechanics in [5, p. 84]. Although the definition given here only applies to the case when $P$ is a cotangent bundle, that is, $P=T^{*} Q$, for some manifold $Q$, it is sufficient for this paper. Corollary 4.2 .11 in [1] guarantees that the definition given here coincides with the general definition when $P=T^{*} Q$.

In the context of a rigid body free to rotate about a fixed point, $\alpha$ is the standard angular momentum of the rigid body. We shall see in the next section that for a rigid body controlled by feedback, the momentum map will be an angular momentum-like quanity - the shifted angular 
momentum. Like angular momentum, in a hamiltonian system, the momentum map is conserved under the flow if the hamiltonian is invariant under the rotation. When the hamiltonian is invariant under an $S O(3)$-action, we say that the hamiltonian system possesses $S O(3)$ - symmetry.

Stability analysis by the energy method finds the equilibria and extremals of the energy functional. For a hamiltonian system with $S O(3)$-symmetry, the momentum map $\alpha$ is conserved under the motion. The dynamic orbit $z(t)$ must satisfy $\boldsymbol{\alpha}(z(t))=\boldsymbol{\alpha}\left(z_{e}\right)$ for an equilibrium point $z_{e}=z(0)$. This is a constrained variation problem. It proves more convenient to remove the constraint by introducing Lagrange multipliers. Thus, we introduce:

Definition 2.5. Let $H$ be the hamiltonian of a hamiltonian system with $S O(3)$-symmetry. Define $H_{\xi}(z):=H(z)-\left\langle\boldsymbol{\alpha}(z)-\boldsymbol{\mu}_{e}, \boldsymbol{\xi}\right\rangle$, where $\boldsymbol{\mu}_{e} \in s o(3)^{*}$ and $\boldsymbol{\xi} \in s o(3)$ are constant. A point $z_{e} \in P$ is called a relative equilibrium, if it is a critical point of $H_{\xi}(z)$ (see [21]). We call $H_{\xi}(z)$ the augmented hamiltonian.

The second term in the expression of $H_{\xi}(z)$ takes into account the fact that $\boldsymbol{\alpha}(z) \equiv \boldsymbol{\mu}_{e}=\boldsymbol{\alpha}\left(z_{e}\right)$ on the flow of $\mathcal{X}_{H}$, with $\xi$ interpreted as a Lagrange multiplier. Here $\mathcal{X}_{H}$ is the vector field of the hamiltonian system.

For the formal stability of a relative equilibrium $z_{e}$, the standard criteria require that the second variation $D^{2} H_{\xi}\left(z_{e}\right)$ be definite. However, due to the invariance of $H$ under the $S O(3)$ action, $z_{e}$ cannot be a strict local extremum. In other words, there are some variations $\delta \mathbf{z}$ such that $D^{2} H_{\xi}\left(z_{e}\right)(\delta \mathbf{z}, \delta \mathbf{z})=0$. These neutral directions must be taken into account. Let $S O(3)_{\mu_{e}}=$ $\left\{\mathbf{A} \in S O(3): \mathbf{A} \boldsymbol{\mu}_{e} \mathbf{A}^{T}=\boldsymbol{\mu}_{e}, \boldsymbol{\mu}_{e}=\boldsymbol{\alpha}\left(z_{e}\right)\right\}$. Denote $\boldsymbol{\mu}_{e}=S\left(\boldsymbol{\pi}_{e}\right)$, then by the identity $: \mathbf{A} S\left(\boldsymbol{\pi}_{e}\right) \mathbf{A}^{T}=$ $S\left(\mathbf{A} \pi_{e}\right)$, one obtains $S O(3)_{\mu_{e}}=\left\{\mathbf{A} \in S O(3): \mathbf{A} \pi_{e}=\pi_{e}\right\}$, i.e., $S O(3)_{\mu_{e}}$ leaves $\pi_{e}$ invariant. In the context of a rotating rigid body, we shall see that the direction of $\pi_{e}$ coincides with the rotation axis at equilibrium. Thus, $S O(3)_{\mu_{e}}=\{\exp (t S(\boldsymbol{\xi})): \boldsymbol{\xi}$ is the axis of uniform rotation, $t \in \mathbf{R}\}$. We can prove (see [21]) that the neutral direction in which $D^{2} H_{\xi}\left(z_{e}\right)(\delta \mathbf{z}, \delta z)=0$ is the tangent direction to the orbit of $z_{e}$ under the $S O(3)_{\mu_{e}}$-action at $z_{e}$. In the present context, it is easily seen $T_{z_{e}}\left(S O(3)_{\mu_{e}} \cdot z_{e}\right)=\left\{\eta_{P}\left(z_{e}\right) \mid \boldsymbol{\eta}\right.$ along rotation axis $\left.\boldsymbol{\xi}\right\}$. In the next section, we shall write down the second variation $D^{2} H_{\xi}\left(z_{e}\right)(\delta z, \delta \mathbf{z})$ explicitly, so that we can clearly see the neutral direction. In general, we shall choose admissible variations $\delta \mathrm{z}$ from a linear subspace $S \subset T_{z_{e}} P$ satisfying $S \cong T_{z_{e}} \boldsymbol{\alpha}^{-1}\left(\boldsymbol{\mu}_{e}\right) / T_{z_{e}}\left(S O(3)_{\mu_{e}} \cdot z_{e}\right)$. We then test the definiteness of $D^{2} H_{\xi}\left(z_{e}\right)(\delta \mathbf{z}, \delta \mathbf{z})$ on $S$. In summary, we have

Definition 2.6. The relative equilibrium $z_{e}$ is said to be formally stable if $D^{2} H_{\xi}\left(z_{e}\right)(\boldsymbol{\delta} \mathbf{z}, \boldsymbol{\delta} \mathbf{z})$ is 
definite for all $\delta \mathbf{z} \in S \cong T_{z_{e}} \boldsymbol{\alpha}^{-1}\left(\boldsymbol{\mu}_{e}\right) / T_{z_{e}}\left(S O(3)_{\mu_{e}} \cdot z_{e}\right)$.

Thus, we can choose $S$ as the complement of $T_{z_{e}}\left(S O(3)_{\mu_{e}} \cdot z_{e}\right)$ in $T_{z_{e}} \boldsymbol{\alpha}^{-1}\left(\boldsymbol{\mu}_{e}\right)$. Formal stability in the context of this paper implies Lyapunov stability on the orbit space $\boldsymbol{\alpha}^{-1}\left(\boldsymbol{\mu}_{e}\right) / S O(3)_{\mu_{e}}$. This will be shown in $\S 5$.

\section{$\S$ 3. Stabilization}

Consider the motion of a rigid body rotating about its center of mass. Let $\Omega \in \mathbb{R}^{3}$ denote the angular velocity in a body-fixed coordinate frame with the origin located at the center of mass of the rigid body. Let $\mathbf{J}$ denote the body-fixed inertia matrix. The motion of the rigid body is then characterized by (cf. [11], [15])

$$
\begin{aligned}
\mathbf{J} \dot{\boldsymbol{\Omega}} & =\mathbf{J} \boldsymbol{\Omega} \times \mathbf{\Omega}+\mathbf{U}, \\
\dot{\mathbf{A}} & =\mathbf{A} S(\boldsymbol{\Omega}),
\end{aligned}
$$

or, in terms of the body angular momentum $\Pi \in \mathbb{R}^{3}$,

$$
\begin{aligned}
& \dot{\mathbf{\Pi}}=\mathbf{\Pi} \times \mathbf{J}^{-1} \boldsymbol{\Pi}+\mathbf{U} \\
& \dot{\mathbf{A}}=\mathbf{A} S(\boldsymbol{\Pi})
\end{aligned}
$$

where $\mathbf{U}=\left(u_{1}, u_{2}, u_{3}\right)^{T}$ is the resultant applied torque; each element $u_{i}$ represents an external torque aligned with the $i$-th body axis. External $u_{i}$ might be generated, for example, by a pair of steering jets. We give the following definition.

Definition 3.1. Given an open loop system $\dot{\mathbf{x}}=f(\mathbf{x}, \mathbf{U})$, a feedback control $\mathbf{U}=\mathbf{g}(\mathbf{x})$ is said to stabilize a point $\mathbf{x}_{e}$, if $\mathbf{x}_{e}$ is a stable equilibrium point of the closed loop system $\dot{\mathbf{x}}=\mathbf{f}(\mathbf{x}, \mathbf{g}(\mathbf{x}))$.

Remark. Here we do not demand that $\mathbf{x}_{e}$ be an equilibrium point of the open loop system. In particular, with regard to $(3.1 \mathrm{a}, \mathrm{b})$, we do not demand that $\Omega_{e}$ be aligned with a principal axis of inertia. In what follows, $\boldsymbol{\Omega}_{e}$ may be along any arbitrary axis.

Consider a family of control laws for $(3.1 \mathrm{a}, \mathrm{b})$ of the form $\mathbf{U}=\mathbf{g}\left(\boldsymbol{\Omega} ; \mathbf{b}_{0}\right)=\boldsymbol{\Omega} \times \mathbf{b}_{0}$, where $\mathbf{b}_{0} \in \mathbb{R}^{3}$ is a constant vector in body coordinates. Under this family of feedback control laws, the closed loop system becomes

$$
\begin{aligned}
\mathbf{J} \dot{\boldsymbol{\Omega}} & =\left(\mathbf{J} \boldsymbol{\Omega}-\mathbf{b}_{0}\right) \times \mathbf{\Omega} \\
\dot{\mathbf{A}} & =\mathbf{A} S(\boldsymbol{\Omega})
\end{aligned}
$$


Our objective is to find conditions on $\mathbf{b}_{0}$ which ensure that $(3.2 \mathrm{a}, \mathrm{b})$ possesses a stable equilibrium corresponding to a given vector $\boldsymbol{\Omega}_{e}$.

Let $\boldsymbol{\Pi}=\mathbf{J} \boldsymbol{\Omega}-\mathbf{b}_{0}$ so that $(3.2 \mathrm{a}, \mathrm{b})$ becomes

$$
\begin{aligned}
& \dot{\mathbf{\Pi}}=\boldsymbol{\Pi} \times \mathbf{J}^{-1}\left(\boldsymbol{\Pi}+\mathbf{b}_{0}\right) \\
& \dot{\mathbf{A}}=\mathbf{A} S\left(\mathbf{J}^{-1}\left(\boldsymbol{\Pi}+\mathbf{b}_{0}\right)\right)
\end{aligned}
$$

Note that $\mathbf{J} \boldsymbol{\Omega}$ is the body angular momentum of the rotating rigid body. We call $\boldsymbol{\Pi}$ the shifted body angular momentum. Let the spatial representation of the shifted angular momentum be denoted by $\boldsymbol{\pi}=\mathbf{A \Pi}$, where $\mathbf{A} \in S O(3)$ is the current configuration of the rigid body. Correspondingly, $\boldsymbol{\omega}=\mathbf{A} \boldsymbol{\Omega}$ is the spatial angular velocity at configuration $\mathbf{A}$. In the present context, the shifted angular momentum $\pi$ coincides with the momentum map introduced in $\S 2.2$ and, hence, is conserved along solutions. This fact can be verified by a direct calculation

$$
\begin{aligned}
\dot{\boldsymbol{\pi}} & =\frac{d(\mathbf{A} \boldsymbol{\Pi})}{d t}=\frac{d \mathbf{A}}{d t} \boldsymbol{\Pi}+\mathbf{A} \frac{d \boldsymbol{\Pi}}{d t}=\mathbf{A} \mathbf{A}^{-1} \frac{d \mathbf{A}}{d t} \boldsymbol{\Pi}+\mathbf{A} \frac{d \boldsymbol{\Pi}}{d t}= \\
& =\mathbf{A}\left(S(\boldsymbol{\Omega}) \boldsymbol{\Pi}+\frac{d \boldsymbol{\Pi}}{d t}\right)=\mathbf{A}\left(\mathbf{J}^{-1}\left(\boldsymbol{\Pi}+\mathbf{b}_{0}\right) \times \mathbf{\Pi}+\frac{d \boldsymbol{\Pi}}{d t}\right) .
\end{aligned}
$$

Substitution of $(3.2 \mathrm{c})$ then shows $\dot{\pi}=\mathbf{0}$.

We have shown

Proposition 3.1. The shifted spatial angular momentum $\pi$ is conserved under the motion.

In body coordinates we have the following.

Corollary 3.1. The norm of $\Pi$ is a constant of the motion.

Proof. Denote the norm of $\boldsymbol{\Pi}$ as $\|\boldsymbol{\Pi}\|$, then $\|\boldsymbol{\Pi}\|^{2}=\|\boldsymbol{\pi}\|^{2}=$ constant.

In spatial coordinates the closed loop system $(3.2 \mathrm{~b})$ can therefore be written as

$$
\begin{aligned}
& \dot{\boldsymbol{\pi}}=0, \\
& \dot{\mathbf{A}}=S(\boldsymbol{\omega}) A
\end{aligned}
$$

where $\mathbf{I}=\mathbf{A} \mathbf{J} \mathbf{A}^{-1}$ is the time-dependent inertia matrix.

Denote the space of spatial shifted angular momenta by $M_{b}$. Since the shifted angular momentum $\boldsymbol{\pi}=\mathbf{I} \boldsymbol{\omega}-\mathbf{b}$, from $\S 2.1, M_{b} \cong s o(3) \cong s o^{*}(3) \cong \mathbb{R}^{3}$. In view of this, we shall denote the elements in $P=T^{*} S O(3)$ by $(\mathbf{A}, S(\boldsymbol{\pi}) \mathbf{A})$, where $\mathbf{A} \in S O(3)$ and $\pi \in M_{b} \cong \mathbf{R}^{3}$. Furthermore, the 
mapping $(\mathbf{A}, S(\boldsymbol{\pi}) \mathbf{A}) \mapsto(\mathbf{A}, \boldsymbol{\pi})$ gives the isomorphism $T^{*} S O(3) \cong S O(3) \times \mathbb{R}^{3}$. Thus, we denote an element in $P=T^{*} S O(3)$ by $(\mathbf{A}, \boldsymbol{\pi})$, where $\boldsymbol{\pi} \in M_{b} \cong \mathbb{R}^{3}$. Consequently, for fixed $z=(\mathbf{A}, \boldsymbol{\pi}) \in P$, an element in $T_{z} P$ can be written as $(\dot{\mathbf{A}}, \dot{\pi} ; z)$. Since $\dot{\mathbf{A}}=S(\boldsymbol{\omega}) \mathbf{A}$ under the same isomorphism as the above, $S(\boldsymbol{\omega}) \mathbf{A} \mapsto \boldsymbol{\omega}$. Thus, $T_{z} P \cong \mathbb{R}^{3} \times \mathbb{R}^{3}$, and $T P \cong P \times \mathbb{R}^{3} \times \mathbb{R}^{3}$. We shall identiy $P$ with $S O(3) \times \mathbb{R}^{3}$ and $T P$ with $P \times \mathbb{R}^{3} \times \mathbb{R}^{3}$. With these identifications, we can now show that the closed loop system (3.3) is hamiltonian. First define the symplectic form (see Appendix A) in the manifold $P=T^{*} S O(3)$ as

$$
\Omega_{z}(\delta \mathbf{z}, \delta \mathbf{z})=\Delta \theta \cdot \delta \pi-\delta \theta \cdot \Delta \pi-\pi \cdot \Delta \theta \times \delta \theta
$$

where $z=(\mathbf{A}, \boldsymbol{\pi}) \in P, \delta \mathbf{z}=(\Delta \boldsymbol{\theta}, \Delta \boldsymbol{\pi} ; z) \in T_{z} P, \boldsymbol{\delta} \mathbf{z}=(\boldsymbol{\delta} \boldsymbol{\theta}, \boldsymbol{\delta} \boldsymbol{\pi} ; z) \in T_{z} P$.

In the symplectic manifold $(P, \Omega)^{1}$, we define the hamiltonian function

$$
H=\frac{1}{2}(\boldsymbol{\pi}+\mathbf{b}) \cdot \mathbf{I}^{-1}(\boldsymbol{\pi}+\mathbf{b})
$$

corresponding to the kinetic energy of the rotating rigid body. In body representation $H=\frac{1}{2}(\Pi+$ $\left.\mathbf{b}_{0}\right) \cdot \mathbf{J}^{-1}\left(\boldsymbol{\Pi}+\mathbf{b}_{0}\right)$, which reflects the invariance of $H$ under the $S O(3)$-action. Thus, we obtain a triple $(P, \Omega, \mathcal{X})$ (see Appendix A), where the vector field $\mathcal{X}$ is characterized by (3.4).

Theorem 3.1. The triple $(P, \Omega, \mathcal{X})$ defined as above is a hamiltonian system.

Proof. A computation shows (see the computation of the first variation of $H$ below) that

$$
D H \cdot \delta \mathbf{z}=\mathbf{I}^{-1}(\boldsymbol{\pi}+\mathbf{b}) \cdot \delta \boldsymbol{\pi}+\mathbf{I}^{-1}(\boldsymbol{\pi}+\mathbf{b}) \times \boldsymbol{\pi} \cdot \delta \boldsymbol{\theta}
$$

where $\mathbf{b}=\mathbf{A} \mathbf{b}_{0}$.

Substituting the vector field $\mathcal{X}$ characterized by (3.4) into the above symplectic form and noticing that the isomorphism $(\mathbf{A}, S(\boldsymbol{\omega}) \mathbf{A}) \mapsto(\mathbf{A}, \boldsymbol{\omega})$ identifies $S(\boldsymbol{\omega}) \mathbf{A}$ with $\boldsymbol{\omega}$, one obtains

$$
\begin{aligned}
\Omega_{z}(\mathcal{X}, \delta \mathbf{z}) & =\omega \cdot \delta \pi-\delta \theta \cdot 0-\pi \times \omega \cdot \delta \theta \\
& =\mathbf{I}^{-1}(\pi+\mathbf{b}) \cdot \delta \pi-\pi \times \mathbf{I}^{-1}(\pi+\mathbf{b}) \cdot \delta \theta \\
& =\mathbf{I}^{-1}(\pi+\mathbf{b}) \cdot \delta \pi+\mathbf{I}^{-1}(\pi+\mathbf{b}) \times \pi \cdot \delta \theta
\end{aligned}
$$

Thus, $\Omega_{z}(\mathcal{X}, \delta \mathbf{z})=D H \cdot \delta \mathbf{z}$, and we conclude that $\mathcal{X}=\mathcal{X}_{H}$ is hamiltonian.

1 Here $\Omega$ stands for the symplectic form rather than angular velocity. 
Remark. The last theorem leads one to ask if there is a phsycial interpretation of the shifted angular momentum $\boldsymbol{\pi}$ and wonder if there is a mechanical extension of the system to a larger system where the closed loop dynamics are realized by an internal torque feedback. Such a mechanical extension does indeed exist - a rigid body carrying three symmetric rotors with associated inertia torques. In fact, the shifted angular momentum $\boldsymbol{\pi}=\mathbf{I} \boldsymbol{\omega}-\mathbf{b}$ corresponds to the total angular momentum in this extended system; I $\boldsymbol{\omega}$ corresponds to the angular momentum with the rotors locked and $-\mathbf{b}$ the angular momentum of the rotor. For further details see [12].

Recall that the augmented hamiltonian is defined as $H_{\xi}(z)=H(z)-\left\langle\boldsymbol{\alpha}(z)-\boldsymbol{\mu}_{e}, \boldsymbol{\xi}\right\rangle$. The momentum map $\boldsymbol{\alpha}$ satisfies $\langle\boldsymbol{\alpha}(q, p), S(\boldsymbol{\xi})\rangle=\left\langle p, S(\boldsymbol{\xi})_{Q}\right\rangle$, for every $S(\boldsymbol{\xi}) \in \operatorname{so}(3),(q, p) \in P=T^{*} Q$. Here the infinitesimal generator associated with $S(\boldsymbol{\xi})$ is computed as $S(\boldsymbol{\xi})_{Q}(\mathbf{A})=\frac{d}{d t} \exp (t S(\boldsymbol{\xi}))$ $\left.\mathbf{A}\right|_{t=0}=S(\boldsymbol{\xi}) \mathbf{A}$. In the present context, $Q=S O(3)$; thus, $q=\mathbf{A} \in S O(3)$ from $\S 2.1, p=$ $S(\boldsymbol{\pi}) \mathbf{A} \in T_{\mathbf{A}}^{*} S O(3)$ and $\langle\boldsymbol{\alpha}(\mathbf{A}, S(\boldsymbol{\pi}) \mathbf{A}), S(\boldsymbol{\xi})\rangle=\left\langle S(\boldsymbol{\pi}) \mathbf{A}, S(\boldsymbol{\xi})_{Q}\right\rangle=\langle S(\boldsymbol{\pi}) \mathbf{A}, S(\boldsymbol{\xi}) \mathbf{A}\rangle=\langle S(\boldsymbol{\pi})$, $S(\boldsymbol{\xi})\rangle$. Thus, $\boldsymbol{\alpha}(\mathbf{A}, S(\boldsymbol{\pi}) \mathbf{A})=S(\boldsymbol{\pi})$. Under the isomorphism $S, \boldsymbol{\alpha}$ is identified with $\boldsymbol{\pi}$, the shifted angular momentum. Note that $\langle\boldsymbol{\alpha}, S(\boldsymbol{\xi})\rangle=\langle S(\boldsymbol{\pi}), S(\boldsymbol{\xi})\rangle=\boldsymbol{\pi} \cdot \boldsymbol{\xi}$. Let $\boldsymbol{\mu}_{e}=S\left(\boldsymbol{\pi}_{e}\right)$, the augmented hamiltonian is then

$$
\begin{aligned}
H_{\xi} & =H-\left\langle\boldsymbol{\alpha}-\boldsymbol{\mu}_{e}, S(\boldsymbol{\xi})\right\rangle, \\
& =H-\left(\boldsymbol{\pi}-\boldsymbol{\pi}_{e}\right) \cdot \boldsymbol{\xi}, \\
& =\frac{1}{2}(\boldsymbol{\pi}+\mathbf{b}) \cdot \mathbf{I}^{-1}(\boldsymbol{\pi}+\mathbf{b})-\left(\boldsymbol{\pi}-\boldsymbol{\pi}_{e}\right) \cdot \boldsymbol{\xi} .
\end{aligned}
$$

Let $z_{e}=\left(\mathbf{A}_{e}, S\left(\boldsymbol{\pi}_{e}\right) \mathbf{A}_{e}\right) \in T^{*} S O(3)$ be a relative equilibrium point of $H_{\xi}$. For any $\boldsymbol{\delta} \boldsymbol{\theta} \in \mathbf{R}^{3}$, we construct the curve

$$
\varepsilon \mapsto \mathbf{A}_{\varepsilon}=\exp (\varepsilon S(\boldsymbol{\delta} \boldsymbol{\theta})) \mathbf{A}_{e} \in S O(3)
$$

passing through $\mathbf{A}_{e}$ at $\varepsilon=0$.

Let $\delta \pi \in \mathbb{R}^{3}$ and consider the curve in $\mathbb{R}^{3}$ defined as

$$
\varepsilon \mapsto \pi_{\varepsilon}+\varepsilon \delta \pi \in \mathbb{R}^{3},
$$

passing through $\pi_{e}$ at $\varepsilon=0$. These constructions induce a curve

$$
\varepsilon \mapsto\left(\mathbf{A}_{\varepsilon}, S\left(\boldsymbol{\pi}_{\varepsilon}\right) \mathbf{A}_{\varepsilon}\right) \in T^{*} S O(3) .
$$

We can now compute the first and second variations of $H_{\xi}$. 
(i) First variation. Set

where

$$
D H_{\xi}\left(z_{e}\right)=\left.\frac{d}{d \varepsilon} H_{\xi, \varepsilon}\right|_{\varepsilon=0}=0
$$

$$
\begin{aligned}
H_{\xi, \varepsilon} & =\frac{1}{2}\left(\boldsymbol{\pi}_{\varepsilon}+\mathbf{b}_{\varepsilon}\right) \cdot \mathbf{I}_{\varepsilon}^{-1}\left(\boldsymbol{\pi}_{\varepsilon}+\mathbf{b}_{\varepsilon}\right)-\boldsymbol{\pi}_{\varepsilon} \cdot \boldsymbol{\xi} \\
\mathbf{I}_{\varepsilon}^{-1} & =\mathbf{A}_{e} \mathbf{J}^{-1} \mathbf{A}_{e}^{-1} \text { and } \mathbf{b}_{e}=\mathbf{A}_{e} \mathbf{b}_{0}
\end{aligned}
$$

At equilibrium we have an additional stationary condition coming from varying the Lagrange multiplier

$$
\left(\boldsymbol{\pi}-\boldsymbol{\pi}_{e}\right) \cdot \boldsymbol{\eta}=0, \text { for all } \boldsymbol{\eta} \in \mathbb{R}^{3} ; \text { i.e., } \boldsymbol{\pi}=\boldsymbol{\pi}_{e} .
$$

After computation analogous to that in [21], we find

$$
\left.\frac{d}{d \varepsilon} H_{\xi, \varepsilon}\right|_{\varepsilon=0}=\delta \boldsymbol{\pi} \cdot\left(\mathbf{I}_{e}^{-1}\left(\boldsymbol{\pi}_{e}+\mathbf{b}_{e}\right)-\boldsymbol{\xi}\right)+\boldsymbol{\delta} \boldsymbol{\theta} \cdot\left(\mathbf{I}_{e}^{-1}\left(\boldsymbol{\pi}_{e}+\mathbf{b}_{e}\right) \times \boldsymbol{\pi}_{e}\right)=0 .
$$

Thus, $\mathbf{I}_{e}^{-1}\left(\boldsymbol{\pi}_{e}+\mathbf{b}_{e}\right)=\boldsymbol{\xi}$ and $\mathbf{I}_{e}^{-1}\left(\boldsymbol{\pi}_{e}+\mathbf{b}_{e}\right) \times \boldsymbol{\pi}_{e}=\mathbf{0}$, from which we conclude $\boldsymbol{\xi} \times \boldsymbol{\pi}_{e}=0$. Assuming $\boldsymbol{\xi} \neq 0$ and $\boldsymbol{\pi}_{e} \neq 0$, we find $\mathbf{I}_{e}^{-1}\left(\boldsymbol{\pi}_{e}+\mathbf{b}_{e}\right)=\lambda^{-1} \boldsymbol{\pi}_{e}$ for some $\lambda \in(-\infty,+\infty)$. In body representation

$$
\boldsymbol{\Omega}_{e} \times \boldsymbol{\Pi}_{e}=\mathbf{0}, \text { and } \mathbf{J}^{-1}\left(\boldsymbol{\Pi}_{e}+\mathbf{b}_{0}\right)=\lambda^{-1} \boldsymbol{\Pi}_{e},
$$

where $\boldsymbol{\Omega}_{e}=\mathbf{A}_{e}^{-1} \boldsymbol{\xi}, \boldsymbol{\Pi}_{e}=\mathbf{A}_{e}^{-1} \boldsymbol{\pi}_{e}$.

Note that at equilibrium the angular velocity and the shift angular momentum are collinear.

(ii) Second variation.

$$
D^{2} H_{\xi}\left(z_{\varepsilon}\right)=\left.\frac{d}{d \varepsilon}\left[\delta \boldsymbol{\pi} \cdot\left(\mathbf{I}_{\varepsilon}^{-1}\left(\boldsymbol{\pi}_{\varepsilon}+\mathbf{b}_{\varepsilon}\right)-\boldsymbol{\xi}\right)+\delta \boldsymbol{\theta} \cdot\left(\mathbf{I}_{\varepsilon}^{-1}\left(\boldsymbol{\pi}_{\varepsilon}+\mathbf{b}_{\varepsilon}\right) \times \boldsymbol{\pi}_{\varepsilon}\right)\right]\right|_{\varepsilon=0}
$$

Once more by computations analogous to those in [21], we obtain the following quadratic form at equilibrium

$$
\begin{aligned}
& D^{2} H_{\xi}\left(z_{e}\right)(\delta \boldsymbol{\pi}, \boldsymbol{\delta} \boldsymbol{\theta})= \\
& \quad\left[\boldsymbol{\delta} \boldsymbol{\pi}^{T} \boldsymbol{\delta} \boldsymbol{\theta}^{T}\right]\left[\begin{array}{cc}
\mathbf{I}_{e}^{-1} & \left(\mathbf{I}_{e}^{-1}-\lambda^{-1} \mathbf{1}_{3}\right) S\left(\boldsymbol{\pi}_{e}\right) \\
-S\left(\boldsymbol{\pi}_{e}\right)\left(\mathbf{I}_{e}^{-1}-\lambda^{-1} \mathbf{1}_{3}\right) & -S\left(\boldsymbol{\pi}_{e}\right)\left(\mathbf{I}_{e}^{-1}-\lambda^{-1} \mathbf{1}_{3}\right) S\left(\boldsymbol{\pi}_{e}\right)
\end{array}\right]\left[\begin{array}{l}
\delta \boldsymbol{\pi} \\
\delta \boldsymbol{\theta}
\end{array}\right] .
\end{aligned}
$$

Now we choose $S$ as follows (see $\S 2.2$ ). First, $S \subset T_{z_{e}} \alpha^{-1}\left(\mu_{e}\right)$, thus, $\delta \alpha=\delta \pi=0$. As we discussed in $\S 2.2$, the neutral direction $T_{z_{e}}\left(G_{\mu_{e}} \cdot z_{e}\right)$ is along the axis of rotation. Restricted to $S$ with $\delta \pi=0$, the second variation becomes

$$
\begin{aligned}
D^{2} H_{\xi}\left(z_{e}\right) & =-\boldsymbol{\delta} \boldsymbol{\theta} \cdot S\left(\boldsymbol{\pi}_{e}\right)\left(\mathbf{I}_{e}^{-1}-\lambda^{-1} \mathbf{1}_{3}\right) S\left(\boldsymbol{\pi}_{e}\right) \boldsymbol{\delta} \boldsymbol{\theta} \\
& =\left(\boldsymbol{\pi}_{e} \times \delta \boldsymbol{\theta}\right) \cdot\left(\mathbf{I}_{e}^{-1}-\lambda^{-1} \mathbf{1}_{3}\right)\left(\boldsymbol{\pi}_{e} \times \boldsymbol{\delta} \boldsymbol{\theta}\right) .
\end{aligned}
$$


Thus, if $\boldsymbol{\delta} \boldsymbol{\theta} \times \boldsymbol{\pi}_{\boldsymbol{e}}=\mathbf{0}$, then $D^{2} H_{\xi}\left(z_{e}\right)(\boldsymbol{\delta} \boldsymbol{\theta}, \boldsymbol{\delta} \boldsymbol{\theta})=0$. Since $\boldsymbol{\pi}_{e}$ and $\boldsymbol{\omega}_{e}$ are collinear (see first variation condition), one sees that the neutral directions are indeed the directions along the rotation axis, as stated in $\S 2.2$. Thus, we choose $S$ to be the orthogonal complement of $\boldsymbol{\omega}_{e}$ in $T_{z_{e}} \boldsymbol{\alpha}^{-1}\left(\boldsymbol{\mu}_{e}\right)$. That is, let $S=\left\{(\delta \boldsymbol{\delta}, \boldsymbol{\delta} \boldsymbol{\theta}) \mid \boldsymbol{\delta} \pi=0, \delta \boldsymbol{\theta} \cdot \boldsymbol{\omega}_{e}=0\right\}$, and test the definiteness of $\left(\mathbf{I}_{e}^{-1}-\lambda^{-1} \mathbf{1}_{3}\right)$ restricted to $S_{0}=\left\{\boldsymbol{\delta} \boldsymbol{\theta} \in \mathbb{R}^{3} \mid \boldsymbol{\delta} \boldsymbol{\theta} \cdot \boldsymbol{\omega}_{e}=0\right\}$. For most applications, the sensors and actuators will be fixed in the rotating coordinate frame of the rigid body. This is also the coordinate frame in which the material properties of the rigid body are naturally formulated. In these coordinates $\delta \Theta=\mathbf{A}_{e}^{-1} \boldsymbol{\delta} \boldsymbol{\theta}, \boldsymbol{\delta} \boldsymbol{\pi}=\mathbf{A}_{e}^{-1} \boldsymbol{\delta} \boldsymbol{\Pi}$ and $\mathbf{J}^{-1}=\mathbf{A}_{e}^{-1} \mathbf{J}^{-1} \mathbf{A}_{e}$. Thus, $D^{2} H_{\xi}\left(z_{e}\right)$ in body representation restricted to $S=\left\{(\delta \Pi, \delta \Theta) \mid \delta \Pi=0, \delta \Theta \cdot \Omega_{e}=0\right\}$ is

$$
D^{2} H_{\xi}\left(z_{e}\right)=\left(\boldsymbol{\Pi}_{e} \times \delta \Theta\right) \cdot\left(\mathbf{J}^{-1}-\lambda^{-1} \mathbf{1}_{3}\right)\left(\boldsymbol{\Pi}_{e} \times \delta \Theta\right) .
$$

Definiteness of $\left(\mathbf{J}^{-1}-\lambda^{-1} \mathbf{1}_{3}\right)$ is then restricted to $S_{0}$. The energy-momentum method thus leads to the following.

Theorem 3.2. The feedback law $\mathbf{U}=\boldsymbol{\Pi} \times \mathbf{J}^{-1} \mathbf{b}_{0}$ stabilizes a nonzero equilibrium $\boldsymbol{\Pi}_{e}$ of (3.2) if and only if there exists a real number $\lambda$, such that

(i) $\mathbf{J}^{-1}\left(\boldsymbol{\Pi}_{e}+\mathbf{b}_{0}\right)=\lambda^{-1} \boldsymbol{\Pi}_{e}$, and

(ii) $\left.\left(\mathbf{J}^{-1}-\lambda^{-1} \mathbf{1}_{3}\right)\right|_{S_{0}}$ is definite.

We can express our results in terms of angular velocity. Recall $\boldsymbol{\Pi}=\mathbf{J} \boldsymbol{\Omega}-\mathbf{b}_{0}$ and $\boldsymbol{\Omega}_{e}$ is a stable equilibrium of (3.4) if and only if $\boldsymbol{\Pi}_{e}=\mathbf{J} \boldsymbol{\Omega}_{e}-\mathbf{b}_{0}$ is a stable equilibrium of (3.2b). Thus, we have the following.

Theorem 3.3. The feedback law $U=\Omega \times b_{0}$ stabilizes a uniform rotation associated with a body angular velocity $\boldsymbol{\Omega}_{e}$, if and only if there exists a real number $\lambda$, such that

(i) $\mathrm{b}_{0}=\mathrm{J} \boldsymbol{\Omega}_{e}-\lambda \boldsymbol{\Omega}_{e}$, and

(ii) $\left.\left(\mathbf{J}^{-1}-\lambda^{-1} \mathbf{1}_{3}\right)\right|_{S_{0}}$ is definite.

Obviously, a sufficient condition for (ii) of Theorems $3.2-3.3$ is that $\left(\mathbf{J}^{-1}-\lambda^{-1} \mathbf{1}_{3}\right)$ be definite. This leads to the following:

Corollary 3.1. $\mathbf{U}=\boldsymbol{\Omega} \times\left(\mathbf{J} \boldsymbol{\Omega}_{e}-\lambda \boldsymbol{\Omega}_{e}\right)$ stabilizes $\boldsymbol{\Omega}_{e} \neq \mathbf{0}$ if $\lambda>\lambda_{\max }(\mathbf{J})$ or $\lambda<\lambda_{\min }(\mathbf{J})$, where $\lambda_{\max }(\mathbf{J})$ and $\lambda_{\min }(\mathbf{J})$ are the largest and smallest eigenvalues of $\mathbf{J}$.

Proof. Let $\mathbf{J}_{P}=\operatorname{diag}\left(\lambda_{1}, \lambda_{2}, \lambda_{3}\right)$, where $\lambda_{i}, i=1,2,3$ are the eigenvalues of $\mathbf{J}$. Observe that $\left(\mathbf{J}_{P}^{-1}-\lambda^{-1} \mathbf{1}_{3}\right)$ and $\left(\mathbf{J}^{-1}-\lambda^{-1} \mathbf{1}_{3}\right)$ have the same characteristic polynomials, therefore, the same eigenvalues. 
Remark. We have found control laws stabilizing an arbitrary angular velocity $\boldsymbol{\Omega}_{e}$. If the rotation is perturbed from $\Omega_{e}$ and the perturbation is not too large, an attitude drift will occur and can be computed by a formula similar to that in [7].

\section{$\S 4$. Reduction of the number of control torques}

In (3.1) - (3.2) of $\S 3, \mathbf{U}=\left(u_{1}, u_{2}, u_{3}\right)^{T}$ is the resultant torque with each $u_{i}$ representing an external torque aligned with $i$-th axis of the body frame. In the case when one or two actuators fail, we have to stabilize the uniform rotation by using the remaining actuators. Moreover, from the theoretical point of view, if all three external torques aligned with coordinate axes available, then the stabilization problem of a uniformly rotating rigid body can be solved by Lyapunov's first method. In fact, in this case, for (3.1) we can design the control law as $\mathbf{U}=-s \mathbf{J}\left(\boldsymbol{\Omega}-\boldsymbol{\Omega}_{e}\right)-\mathbf{J} \boldsymbol{\Omega}_{e} \times \boldsymbol{\Omega}_{e}$, where $s$ is a positive number. The closed loop system is then

$$
J \dot{\boldsymbol{\Omega}},=\mathbf{J} \boldsymbol{\Omega} \times \boldsymbol{\Omega}-s \mathbf{J}\left(\boldsymbol{\Omega}-\boldsymbol{\Omega}_{e}\right)-\mathbf{J} \boldsymbol{\Omega}_{e} \times \boldsymbol{\Omega}_{e}
$$

or

$$
\dot{\boldsymbol{\Omega}}=\mathbf{J}^{-1}(\mathbf{J} \boldsymbol{\Omega} \times \boldsymbol{\Omega})-s\left(\boldsymbol{\Omega}-\boldsymbol{\Omega}_{e}\right)-\mathbf{J}^{-1}\left(\mathbf{J} \boldsymbol{\Omega}_{e} \times \boldsymbol{\Omega}_{e}\right) .
$$

Linearizing (4.1b) at $\Omega_{e}$ gives

$$
\dot{\boldsymbol{\Omega}}=\left(\mathbf{F}-s \mathbf{1}_{3}\right) \boldsymbol{\Omega}
$$

where $\mathbf{F}$ is the Jacobi matrix of $\mathbf{J}^{-1}(\mathbf{J} \boldsymbol{\Omega} \times \boldsymbol{\Omega})$ evaluated at $\boldsymbol{\Omega}_{e}$. It can easily be proved that for large enough $s,\left(\mathbf{F}-s \mathbf{1}_{3}\right)$ is a stable matrix. According to Lyapunov's first method (see [15], Theorem 10.1), (4.1a) will be stable at $\boldsymbol{\Omega}_{e}$ for large enough $s$. Thus, $\mathbf{U}=-s \mathbf{J}\left(\boldsymbol{\Omega}-\boldsymbol{\Omega}_{e}\right)-\mathbf{J} \boldsymbol{\Omega}_{e} \times \boldsymbol{\Omega}_{e},(s \gg 1)$ stabilizes $\Omega_{e}$ for the original system (3.1). However, this method will not be applicable in either of the following two situations: (i) the number of available control torques are less than three, (ii) a flexible appendage is attached. Therefore, the reduction of the number of control torques is of both practical and theoretical interest. Previous results about stabilizing zero angular velocity by one or two torques have been reported in, for example, [2] and [8]. In [6] and [7] only one torque was used to stabilize rotation about the intermediate principal axis. In [25] results related to stabilizing an angular velocity by using two torques were reported. In this section we investigate the problem of stabilizing an angular velocity by one torque. In general, rotation about an arbitrary axis cannot be stabilized with only one torque aligned with a coordinate axis. However, if the coordinate frame has been chosen so that the axis of rotation lies on the $X-Z$ plane and the inertia matrix of the 
rigid body under this frame has the form

$$
\mathbf{J}=\left[\begin{array}{ccc}
j_{11} & 0 & j_{13} \\
0 & j_{22} & 0 \\
j_{13} & 0 & j_{33}
\end{array}\right]
$$

then stabilizing feedback control laws with only one torque aligned with $Y$-axis can be obtained. Theoretically a coordinate frame can always be chosen to coincide with the principal-axis frame, in which case the inertia matrix has the above form with $j_{13}=0$. However, in practical applications we have to choose a coordinate frame convenient for measurement and computation. Fortunately, in many cases there are convenient and physically motivated coordinate frames, in which the inertia matrix of the rigid body has this form. Typically, if the shape and mass distribution of the rigid body is symmetric with respect to reflection in a plane, then its center of mass and two of its principal axes must lie in that plane, the remaining principal axis being perpendicular to it (see [19], p. 173-174). If a coordinate frame has the $Y$-axis aligned with the remaining princpal axis, then it is easily verified (see [19], p. 173-174) that the inertia matrix of the rigid body under this frame has the desired form (4.3).

We shall see in $\S 5$ that the inertia matrix of a space shuttle has this form. Furthermore, if the axis of rotation lies on the plane of symmetry, then from the results in $\S 3$, we can derive new stabilizing control laws in which only one applied torque is used. These control laws are presented in the following two corollaries.

Corollary 4.1. Let $\mathbf{J}$ have the form (4.3) and $\boldsymbol{\Omega}=\left(\omega_{1}, \omega_{2}, \omega_{3}\right)^{T}$. The control $\mathbf{U}=\left(0, u_{2}, 0\right)^{T}$ then stabilizes $\boldsymbol{\Omega}_{e}=\left(w_{1}, 0, w_{3}\right)^{T}$, where

$$
u_{2}=\frac{\left(\left(\lambda-j_{11}\right)\left(j_{22}-j_{33}\right)-j_{13}^{2}\right) w_{1} \omega_{3}+\left(\left(\lambda-j_{33}\right)\left(j_{11}-j_{22}\right)+j_{13}^{2}\right) w_{3} \omega_{1}}{\lambda-j_{22}}+j_{13}\left(w_{3} \omega_{3}-w_{1} \omega_{1}\right)
$$

with $\lambda>\lambda_{\max }(\mathbf{J})$ or $\lambda<\lambda_{\min }(\mathbf{J})$. Where $\lambda_{\max }(\mathbf{J})$ and $\lambda_{\min }(\mathbf{J})$ are respectively the largest and smallest eigenvalues of $\mathbf{J}$. 
Proof. The idea of the proof is the following: We do a variable transformation by adding a parameter vector $\Omega_{0}$ to $\Omega_{e}$. Then, using the results in $\S 3$, we can stabilize the new sytem and get a control law containing $\boldsymbol{\Omega}_{0}$. By transforming back to the original variables, we then get a control law stabilizing the original system. This control law containing $\Omega_{0}$. By choosing $\Omega_{0}$ properly, we can make two components of this control become zero. To be specific, let $\Omega_{0}=(a, b, c)^{T}$ be a constant parameter vector to be determined and set $\Omega^{*}=\boldsymbol{\Omega}+\boldsymbol{\Omega}_{0}$. By Corollary 3.1, $\boldsymbol{\Omega}_{e}^{*}=\boldsymbol{\Omega}_{e}+\boldsymbol{\Omega}_{0}$ is then a stable equilibrium of

$$
\mathbf{J} \dot{\boldsymbol{\Omega}}^{*}=\left(\mathbf{J} \boldsymbol{\Omega}^{*}-\left(\mathbf{J} \boldsymbol{\Omega}_{e}^{*}-\lambda \dot{\boldsymbol{\Omega}}_{e}^{*}\right) \times \boldsymbol{\Omega}^{*}\right.
$$

That is, $\boldsymbol{\Omega}_{e}$ is a stable equilibrium of

$$
\mathbf{J} \dot{\boldsymbol{\Omega}}=\mathbf{J} \boldsymbol{\Omega} \times \boldsymbol{\Omega}+\left[\left(\mathrm{J} \boldsymbol{\Omega}-\mathbf{J} \boldsymbol{\Omega}_{e}+\lambda \boldsymbol{\Omega}_{e}\right) \times \boldsymbol{\Omega}_{0}-\left(\mathrm{J} \boldsymbol{\Omega}_{e}-\lambda \boldsymbol{\Omega}_{e}-\lambda \boldsymbol{\Omega}_{0}\right) \times \boldsymbol{\Omega}\right]
$$

The feedback control law stabilizing (3.1) corresponds to the term inside the square backet. Specifically, we have

$$
\begin{aligned}
\mathbf{U} & =\left(\mathbf{J} \boldsymbol{\Omega}-\mathbf{J} \boldsymbol{\Omega}_{e}+\lambda \boldsymbol{\Omega}_{e}\right) \times \boldsymbol{\Omega}_{0}-\left(\mathbf{J} \boldsymbol{\Omega}_{e}-\lambda \boldsymbol{\Omega}_{e}-\lambda \boldsymbol{\Omega}_{0}\right) \times \boldsymbol{\Omega} \\
& =\left(u_{1}, u_{2}, u_{3}\right)^{T},
\end{aligned}
$$

where $u_{1}, u_{2}$ and $u_{3}$ are functions of $\Omega_{0}=(a, b, c)^{T}$. Letting $b=0, u_{1}=u_{3}=0$ and solving for $a$ and $c$, one obtains

$$
a=\frac{j_{13} w_{3}-\left(\lambda-j_{11}\right) w_{1}}{\lambda-j_{22}}, \quad c=\frac{j_{13} w_{1}-\left(\lambda-j_{33}\right) w_{3}}{\lambda-j_{22}}
$$

Substituting $\boldsymbol{\Omega}_{0}=(a, b, c)^{T}$ into $\mathbf{U}$ establishes the result.

This control law can be further simplified:

Corollary 4.2. Let $\mathbf{J}$ have the form in (4.3) and $\boldsymbol{\Omega}=\left(\omega_{1}, \omega_{2}, \omega_{3}\right)^{T}$. Then

$$
\mathbf{U}=\left(0,\left(\left(j_{11}-j_{22}\right) w_{3}-j_{13} w_{1}\right) \omega_{1}+\left(\left(j_{22}-j_{33}\right) w_{1}+j_{13} w_{3}\right) \omega_{3}, 0\right)^{T}
$$

stabilizes $\boldsymbol{\Omega}_{e}=\left(w_{1}, 0, w_{3}\right)^{T}$.

Proof. In corollary 4.1 let $\lambda \rightarrow \infty$. The control law described there then tends to the control law here. The limit process can be justified by using the theorem of differential equations about the continuity of solution depending on parameters and the fact that the solutions are periodic (cf. $[17])$. 


\section{§5. Examples}

Example 1. Consider stabilizing the uniform rotation of a space shuttle. Assume a body frame with the origin fixed at the center of mass of the space shuttle. For this coordinate frame the $X$-axis is along the centroid of the cargo bay, the $Z$-axis point up out of the cargo bay and the $Y$-axis completes a right-handed coordinate system. In this coordinate system the following body-fixed inertia matrix has the form (4.3). Clearly the $Y$-axis is along one of the principal axes of inertia (the intermediate axis), while the $X$-axis and $Z$-axis are not.

For this example we have scaled the data found in [24]. The nonzero values of $\mathbf{J}$ are $j_{11}=$ $9.05443, j_{13}=-1.45393, j_{22}=67.891$ and $j_{33}=70.86601$. A simple calculation will verify that the $Y$-axis is the intermediate axis of inertia. We seek to stabilize the uniform rotation corresponding to an angular velocity $\Omega_{e}=(0,10.0,0)^{T}$. From Corollary 3.1 we have the control law $\mathbf{U}=\left(121.09 \omega_{3}, 0,-121.09 \omega_{1}\right)^{T}$ corresponding to uniform rotation about the (non-principal) pitch axis. For our data, figure 1 illustrates the time response of this control law. The solid curves represent the three components of angular velocity of the open loop system with a perturbed initial value $\Omega(0)=(0.01,10.01,0.01)^{T}$. These curves show that the motion is unstable and the rigid body is tumbling. The straight, dashed and dotted lines are the three components of angular velocity of the controlled system with the same perturbed initial value $\Omega(0)$. The "straight" lines show that the uniform rotation has been stabilized by the control.

Example 2. Consider a rigid body with inertia matrix $\mathbf{J}=\operatorname{diag}(4,6,8)$. We want to stabilize the rotation about $\boldsymbol{\Omega}_{e}=(3,6,0)^{T}$. Methods 1 and 2 find the control laws stabilizing $\boldsymbol{\Omega}_{e}=(3,6,0)^{T}$, by using Corollary 3.1 and 4.1 , respectively.

Method 1: From Corollary 3.1, we have the following control law (set $\lambda=10$ ):

$$
\mathbf{U}=\left(24 \omega_{3} 2,-18 \omega_{3},-24 \omega_{1}+18 \omega_{2}\right)^{T}
$$

Figure 2 shows that this control law stabilizes $\boldsymbol{\Omega}_{e}=(3,6,0)^{T}$.

Method 2: From Corollary 4.1, we have the following control law (set $\lambda=20$ ):

$$
\mathbf{U}=\left(0,0,28 \omega_{1},-8 \omega_{2}\right)^{T}
$$

Figure 3 shows that this control law stabilizes $\boldsymbol{\Omega}_{e}=(3,6,0)^{T}$. 


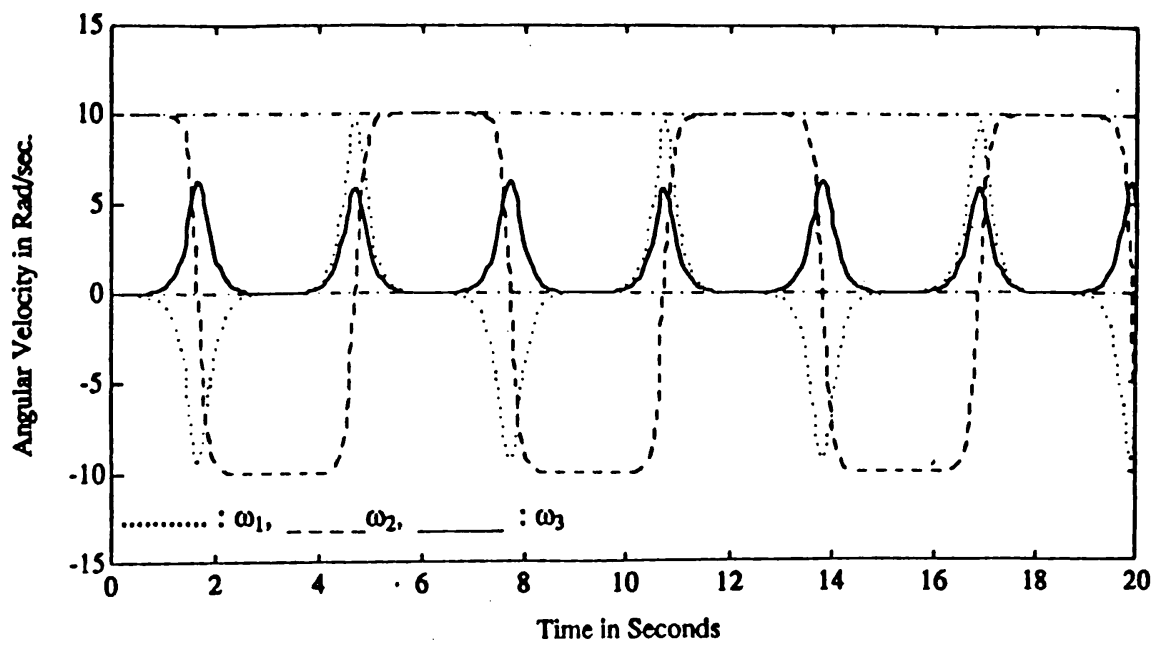

Figure 1. Time response for example 1.

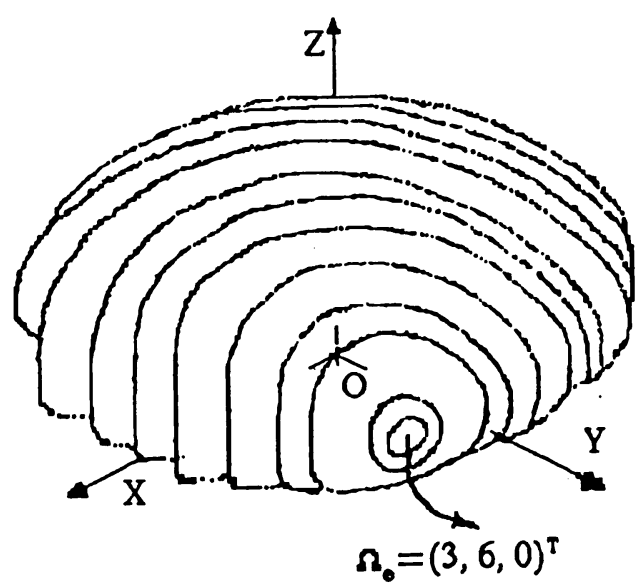

Figure 2. Phase flow for example 2 using first method.

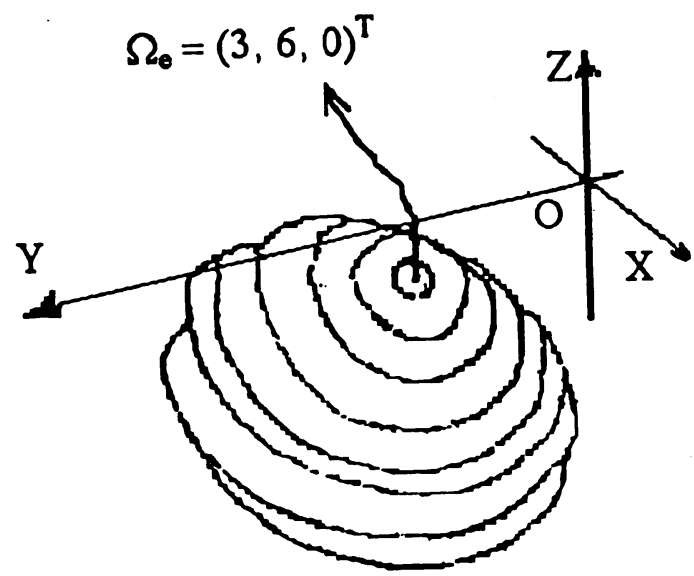

Figure 3. Phase flow for example 2 using second method. 


\section{§6. Robust Stability}

In this section, we shall define the robustness of a family of control laws, then prove that the control law presented in Corollary 3.1 is robust. This section is divided into two parts: The first, $\S 6.1$, can be viewed as a continuation of $\S 2.2$ and introduces material needed in $\S 6.2$. In $\S 6.2$ we discuss the issue of robustness.

\section{$\S 6.1$. Reduced space and stability}

In $\S 2.2$, we defined the concept of formal stabilty. In this subsection, we explore the relationship between formal stability and Lyapunov stability. We shall prove that formal stability of the spatial shifted angular momentum $\pi_{e}$ implies Lyapunov stability of body shifted angular momentum $\Pi_{e}$. From Proposition 3.2, the trajectory of the body shifted angular momentum stays on a sphere with dimension two. The original phase space $P=T^{*} S O(3)$ has six dimensions. Thus, we call the two-dimensional sphere the reduced space of $P$. The reduced space can also be defined in a different way. Recall that in $\S 2.2$, we defined the isotropy group as $S O(3)_{\mu}=\left\{\mathbf{A} \in S O(3): \mathbf{A} \boldsymbol{\mu} \mathbf{A}^{-1}=\boldsymbol{\mu}, \boldsymbol{\mu}=\boldsymbol{\alpha}(z)\right\}$ and introduced the concept of group orbit. If we consider $S O(3)_{\mu}$ as a group acting on $\alpha^{-1}(\boldsymbol{\mu})$, then a more general, alternative definition of reduced space is as follows (cf. [1], [4] and [14]).

Definition 6.1. The set of orbits $\alpha^{-1}(\boldsymbol{\mu}) / S O(3)_{\mu}$ is called the reduced space of $P$ and denoted as $P_{\mu}$. Each orbit in $P$ is a point in space $P_{\mu}$.

The following Lemma shows that the above definition is indeed equivalent to what we have just said.

Lemma 6.1. Let $\boldsymbol{\mu}=S(\boldsymbol{\pi})$, then $P_{\mu} \cong \partial B(0,\|\boldsymbol{\pi}\|):=\left\{\boldsymbol{\Pi} \in \mathbb{R}^{3} \mid\|\boldsymbol{\Pi}\|=\|\boldsymbol{\pi}\|\right\}$.

Proof. Let $z=(\mathbf{A}, S(\boldsymbol{\pi}) \mathbf{A}) \in \boldsymbol{\alpha}^{-1}(\boldsymbol{\mu})$, where $\boldsymbol{\mu}=S(\boldsymbol{\pi}), \mathbf{A} \in S O(3)$. Define a mapping $\boldsymbol{\varphi}: P_{\boldsymbol{\mu}} \rightarrow$ $\partial \mathbf{B}(0,\|\boldsymbol{\pi}\|)$, by $\varphi([z])=\mathbf{A}^{-1} \boldsymbol{\pi}$, where $[z] \in P_{\mu}$ is the $S O(3)_{\mu}$-orbit of $z$. Note that for $z^{\prime} \in[z]$, i.e., $z^{\prime}=\mathbf{B} \cdot z=(\mathbf{B A}, S(\boldsymbol{\pi}) \mathbf{B A})$, for some $\mathbf{B} \in S O(3)_{\mu}, \varphi\left(\left[z^{\prime}\right]\right)=(\mathbf{B A})^{-1} \boldsymbol{\pi}=\mathbf{A}^{-1} \mathbf{B}^{-1} \boldsymbol{\pi}=\mathbf{A}^{-1} \boldsymbol{\pi}=$ $\varphi([z])$. Thus, $\varphi$ is well defined. Moreover, $\varphi$ is a diffeomorphism (cf. [1]).

Given a $S O(3)$-invariant hamiltonian $H$ on $P, H$ induces a reduced hamiltonian on the reduced space $P_{\mu}$ in the following way:

Definition 6.2. Let $H$ be a $G$-invariant hamiltonian on $P$, then $H^{\mu}$ is defined by

$$
H^{\mu}([z])=H(z) \text { for all } z \in \boldsymbol{\alpha}^{-1}(\boldsymbol{\mu}) \subset P,
$$


where $[z] \in P_{\mu}$ is the $S O(3)_{\mu}$-orbit of $z$. We call $H^{\mu}$ the reduced hamiltonian on $P_{\mu}$. Note that since $H$ is $S O(3)$-invariant, for $z^{\prime} \in[z], H\left(z^{\prime}\right)=H(z)$. Hence, $H^{\mu}$ is well defined. The reason that $H^{\mu}$ is hamiltonian is that we can also induce a symplectic form $\Omega_{\mu}$ on the reduced space $P_{\mu}$, in which case $\left(P_{\mu}, \Omega_{\mu}, H^{\mu}\right)$ becomes a hamiltonian system (cf. [1], [4] and [14]).

Corollary 6.1. If $\boldsymbol{\pi}_{e}$ is formally stable, then $\Pi_{e}$ is a conditional, strict, local extremum point of the hamiltonian $H^{\mu}=\frac{1}{2}\left(\boldsymbol{\Pi}+\mathbf{b}_{0}\right) \cdot \mathbf{J}^{-1}\left(\mathbf{\Pi}+\mathbf{b}_{0}\right)$ on the sphere

$$
\partial B\left(\mathbf{0},\left\|\boldsymbol{\Pi}_{e}\right\|\right):=\left\{\boldsymbol{\Pi} \in \mathbb{R}^{3} \mid\|\boldsymbol{\Pi}\|=\left\|\boldsymbol{\Pi}_{e}\right\|\right\}
$$

Proof. By definition 2.6, for all $\delta \mathbf{z} \in S \cong T_{z_{e}} \boldsymbol{\alpha}^{-1}\left(\pi_{e}\right) / T_{z_{e}} S O(3)_{\mu_{e}} \cdot z_{e}$ the second variation $\delta^{2} H_{\xi}\left(z_{e}\right)(\delta \mathbf{z}, \delta \mathbf{z})$ is definite. Thus, $z_{e}=\left(\mathbf{A}_{e},\left[\boldsymbol{\pi}_{e}\right] \mathbf{A}_{e}\right)$ (more precisely, $\left.z_{e}=\left(\mathbf{A}_{e}, \boldsymbol{\pi}_{e}\right)\right)$. is the strict extremum point of the reduced hamiltonian $H^{\mu}$ on $P_{\mu}$. However, $\boldsymbol{\Pi}_{e}=\mathbf{A}_{e} \boldsymbol{\pi}_{e}, H^{\mu}\left(\left[z_{e}\right]\right)=\frac{1}{2}(\boldsymbol{\Pi}+$ $\left.\mathbf{b}_{0}\right) \cdot \mathbf{J}^{-1}\left(\boldsymbol{\Pi}+\mathbf{b}_{0}\right)$, and $P_{\mu}=\partial B\left(0,\left\|\boldsymbol{\Pi}_{e}\right\|\right)$, by Lemma 6.1. Thus, the assertion follows.

The following is the main theorem of this subsection. The proof is based on a similar result in $[20$, p. 208] and contains results which we will use subsequently.

Theorem 6.1. Suppose that $\pi_{e}$ is a relative equilibrium and is formally stable, then $\Pi_{e}$ is a Lyapunov stable equilibrium (3.4), the same statement holds for $\boldsymbol{\omega}_{e}, \boldsymbol{\Omega}_{\boldsymbol{e}}$ and (3.3).

Proof. By Corollary 6.1., $\boldsymbol{\Pi}_{e}$ is a strict, conditional extremum point of $H^{\mu}(\boldsymbol{\Pi}):=\frac{1}{2}\left(\boldsymbol{\Pi}+\mathbf{b}_{0}\right)$. $\mathbf{J}^{-1}\left(\boldsymbol{\Pi}+\mathbf{b}_{0}\right)$ on the sphere $\partial B\left(\mathbf{0},\left\|\boldsymbol{\Pi}_{e}\right\|\right):=\left\{\boldsymbol{\Pi} \in \mathbb{R}^{3} \mid\|\boldsymbol{\Pi}\|=\left\|\boldsymbol{\Pi}_{e}\right\|\right\}$. Without loss of generality, assume $\Pi_{e}$ is a minimum point. If not, replace $H^{\mu}(\Pi)$ by $-H^{\mu}(\Pi)$.

Define $H^{*}(\boldsymbol{\Pi})=H^{\mu}(\boldsymbol{\Pi})-\frac{\lambda}{2}\left(\|\boldsymbol{\Pi}\|^{2}=\left\|\boldsymbol{\Pi}_{e}\right\|^{2}\right)$. Observe that

(i) $H^{*}(\mathbf{\Pi})=H^{\mu}(\boldsymbol{\Pi})$, for $\boldsymbol{\Pi} \in \partial B\left(\mathbf{0},\left\|\mathbf{\Pi}_{e}\right\|\right)$;

(ii) Since $H^{\mu}(\boldsymbol{\Pi})$ and $\|\mathbf{\Pi}\|$ are conserved along the flow, so is $H^{*}(\boldsymbol{\Pi})$.

For an arbitrarily small given $\varepsilon>0$, let $\bar{B}\left(\boldsymbol{\Pi}_{e}, \varepsilon\right)=\left\{\mathbf{x} \in \mathbb{R}^{3} \mid\left\|\mathbf{x}-\boldsymbol{\Pi}_{e}\right\| \leq \varepsilon\right\}$ and 
$\partial B\left(\boldsymbol{\Pi}_{e}, \varepsilon\right)=\left\{\mathbf{x} \in \mathbb{R}^{3} \mid\left\|\mathbf{x}-\boldsymbol{\Pi}_{e}\right\|=\varepsilon\right\}$. Define $F(\varepsilon)=\left\{\boldsymbol{\Pi} \in \bar{B}\left(\boldsymbol{\Pi}_{e}, \varepsilon\right) \mid H^{*}(\boldsymbol{\Pi})<h_{\min }(\varepsilon)\right\}$, where $h_{\min }(\varepsilon)=\min \left\{H^{*}(\boldsymbol{\Pi}): \Pi \in \partial B\left(\boldsymbol{\Pi}_{e}, \varepsilon\right)\right\}$. Since $\boldsymbol{\Pi}_{e}$ is a conditional (strict) extremum point of $H^{\mu}(\boldsymbol{\Pi})$ on the sphere $\partial B\left(\mathbf{0},\left\|\boldsymbol{\Pi}_{e}\right\|\right)$, it is easily seen that $\boldsymbol{\Pi}_{e}$ is the absolute (strict) extremum of $H^{*}(\boldsymbol{\Pi})$. Thus, $H^{*}(\boldsymbol{\Pi})<h_{\min }(\varepsilon)$. That is, $\boldsymbol{\Pi}_{e} \in F(\varepsilon)$. Note that $F(\varepsilon)$ is an open set containing $\boldsymbol{\Pi}_{e}$, thus, $F(\varepsilon)$ contains open balls $\left\{B\left(\boldsymbol{\Pi}_{e}, r_{p}\right)\right\} p \in T$ centered at $\boldsymbol{\Pi}_{e}$ with radius $\left\{r_{p}\right\}, p \in T$, where $T$ is an index set. Let $\delta=\sup \left\{r_{p} \mid p \in T\right\}$. Note that $0<\delta \leq \varepsilon$, since $0<r_{p}<\varepsilon$, for all $p \in T$.

We claim that $\left\|\boldsymbol{\Pi}(t)-\boldsymbol{\Pi}_{e}\right\|<\varepsilon$, for all $t \geq 0$, as $\left\|\boldsymbol{\Pi}(0)-\boldsymbol{\Pi}_{e}\right\|<\delta$. In fact, $\left\|\boldsymbol{\Pi}(0)-\boldsymbol{\Pi}_{e}\right\|<\delta$ implies $\Pi(0) \in F(\varepsilon) \Rightarrow H^{*}(\Pi(0))<h_{\min }(\varepsilon)$. On the other hand, since $H^{*}(\Pi)$ is conserved along the flow, $H^{*}(\boldsymbol{\Pi}(t))<h_{\min }(\varepsilon)$ for all $t>0$. Now, if $\left\|\boldsymbol{\Pi}(t)-\boldsymbol{\Pi}_{e}\right\| \geq \varepsilon$ for some $t>0$, then since $\left\|\boldsymbol{\Pi}(0)-\Pi_{e}\right\|<\varepsilon$, there exists a $t_{0}>0$ such that $\left\|\Pi\left(t_{0}\right)-\Pi_{e}\right\|=\varepsilon$ by the continuity of $\Pi(t)$. Thus, by the definition of $h_{\min }(\varepsilon), H^{*}\left(\Pi\left(t_{0}\right)\right) \geq h_{\min }(\varepsilon)$, which contradicts the fact that $H^{*}(\boldsymbol{\Pi}(t))<h_{\min }(\varepsilon)$ for all $t \geq 0$. Hence, $\left\|\mathbf{\Pi}(t)-\Pi_{e}\right\|<\varepsilon$ for all $t \geq 0$. Thus, we conclude that $\Pi_{e}$ is Lyapunov stable.

Now, consider $\boldsymbol{\Omega}(t)=\mathbf{J}^{-1}\left(\boldsymbol{\Pi}+\mathbf{b}_{0}\right)$. Observe that $\underline{\sigma}\left(\mathbf{J}^{-1}\right)\left\|\boldsymbol{\Pi}(t)-\boldsymbol{\Pi}_{e}\right\| \leq\left\|\boldsymbol{\Omega}(t)-\boldsymbol{\Omega}_{e}\right\|=$ $\left\|\mathbf{J}^{-1}\left(\boldsymbol{\Pi}(t)-\boldsymbol{\Pi}_{e}\right)\right\| \leq \bar{\sigma}\left(\mathbf{J}^{-1}\right)\left\|\boldsymbol{\Pi}(t)-\boldsymbol{\Pi}_{e}\right\|$, where $\underline{\sigma}\left(\mathbf{J}^{-1}\right)$ and $\bar{\sigma}\left(\mathbf{J}^{-1}\right)$ denote the largest and smallest singular values of $\mathbf{J}^{-1}$. Assume $\mathbf{J}$ has eigenvalues $j_{1} \leq j_{2} \leq j_{3}$. Then, $j_{1}^{-1}\left\|\mathbf{\Pi}(t)-\boldsymbol{\Pi}_{e}\right\| \leq$ $\left\|\boldsymbol{\Omega}(t)-\boldsymbol{\Omega}_{e}\right\| \leq j_{3}^{-1}\left\|\boldsymbol{\Pi}(t)-\boldsymbol{\Pi}_{e}\right\|$. For a given $\varepsilon>0$, let $\varepsilon^{*}=\varepsilon j_{3}$. By the above analysis, there must exist a $\delta^{*}>0$, such that $\left\|\boldsymbol{\Pi}(t)-\boldsymbol{\Pi}_{e}\right\|<\varepsilon^{*}$ for all $t \geq 0$, as $\left\|\boldsymbol{\Pi}(0)-\Pi_{e}\right\|<\delta^{*}$. Taking $\delta=\delta^{*} j_{1}^{-1}$, we find $\left\|\boldsymbol{\Omega}(t)-\boldsymbol{\Omega}_{e}\right\| \leq \varepsilon$ for $t \geq 0$, as $\left\|\boldsymbol{\Omega}(0)-\boldsymbol{\Omega}_{e}\right\| \leq \delta$.

The magnitude of $\delta$ introduced in this proof is a measure of stability. A formula for the computation of $\delta$ is given by the following: 
Proposition 6.1. Suppose that the inertia matrix $\mathbf{J}$ has distinct eigenvalues $\left\{j_{1}, j_{2}, j_{3}\right\}$. Assume these are ordered such that $j_{1}>j_{2}>j_{3}$. Then for the closed loop system in (3.3),

$$
\delta=\left\{\begin{array}{l}
\frac{j_{3}}{j_{1}} \sqrt{\frac{j_{3}\left(\lambda-j_{1}\right)}{j_{1}\left(\lambda-j_{3}\right)}}, \varepsilon \text { for } \lambda>j_{1} \\
\frac{j_{3}}{j_{1}} \sqrt{\frac{j_{1}\left(j_{3}-\lambda\right)}{j_{3}\left(j_{1}-\lambda\right)},}, \varepsilon \text { for } \lambda<j_{3}
\end{array}\right.
$$

where $\delta$ is defined as in the proof of Theorem 6.1 .

Proof. See Appendix 2.

Corollary 6.2. Assume $\delta(\lambda, \mathbf{J})$ is defined as above. Then, for an arbitrary inertia matrix $\mathbf{J}$ having eigenvalues $\left\{j_{1}, j_{2}, j_{3}\right\}, j_{1}>j_{2}>j_{3}$, the following statements hold:

(i) $\delta(\lambda, \mathbf{J})$ is a strictly increasing function of $|\lambda|$ for $\lambda \in\left(j_{1},+\infty\right)$ and for $\lambda \in(-\infty, 0)$;

(ii) $\delta(\lambda, \mathbf{J})$ is a continuous function of the entries of $\mathbf{J}$ over a certain closed set, for $\lambda>j_{1}$ or $\lambda<j_{3}$

Proof. Simple algebraic computation verifies (i). The second part of the conclusion comes from the fact that the eigenvalues of $\mathbf{J}$ are continuous functions of the entries of $\mathbf{J}$, and for $\lambda>j_{1}$ or $\lambda<j_{3}$, the denominators in $\delta$ are nonzero.

Remark. The scalar $\delta$ can be viewed as a measure of the degree of stability: A motion with large $\delta$ is "more stable" than that with small $\delta$. Comment (i) says that the increase of $|\lambda|$ leads to the increase of the degree of stability. Comment (ii) says that the degree of stability $\delta$ changes gradually as $\mathbf{J}$ changes. Thus, increasing $|\lambda|$ can compensate for the loss of degree of stability casued by the change in $\mathbf{J}$.

\section{§6.2. Robust stability}

We first show how uncertainty in the inertia matrix $\mathbf{J}$ may cause the control law to fail. Consider stabilization of the body angular velocity $\boldsymbol{\Omega}_{e}$ for Euler's equation $\mathbf{J} \boldsymbol{\Omega}=\mathbf{J} \boldsymbol{\Omega} \times \boldsymbol{\Omega}+\mathbf{U}$ 
by a feedback control law $\mathbf{U}=\mathbf{f}(\boldsymbol{\Omega})$. In other words, find a $\mathbf{U}=\mathbf{f}(\boldsymbol{\Omega})$ such that $\boldsymbol{\Omega}_{e}$ is a stable equilibrium point of the closed loop system $\mathbf{J} \dot{\boldsymbol{\Omega}}=\mathbf{J} \boldsymbol{\Omega} \times \boldsymbol{\Omega}+\mathbf{f}(\boldsymbol{\Omega})$. A number of methods for stabilizing the null solution $\left(\Omega_{e}=0\right)$ of Euler's equation have appeared in the literature. One might consider generalizing them to the problem of stabilizing an arbitrary $\boldsymbol{\Omega}_{e}$ as follows: Assume $\mathbf{U}=\mathbf{f}_{0}\left(\boldsymbol{\Omega}_{0}\right)$ is a feedback control law which stabilizes the null solution of $\mathbf{J} \dot{\boldsymbol{\Omega}}_{0}=\mathbf{J} \boldsymbol{\Omega}_{0} \times \boldsymbol{\Omega}_{0}$. Letting $\dot{\Omega}_{0}=\Omega-\Omega_{e}$ the closed loop dynamics are

$$
\mathbf{J} \dot{\boldsymbol{\Omega}}=\mathbf{J}\left(\boldsymbol{\Omega}-\boldsymbol{\Omega}_{e}\right) \times\left(\boldsymbol{\Omega}-\boldsymbol{\Omega}_{e}\right)+\mathrm{f}_{0}\left(\boldsymbol{\Omega}-\boldsymbol{\Omega}_{e}\right) .
$$

After rearranging

$$
\mathrm{J} \dot{\boldsymbol{\Omega}}=\mathbf{J} \boldsymbol{\Omega} \times \boldsymbol{\Omega}+\left\{\mathrm{J} \boldsymbol{\Omega}_{e} \times\left(\boldsymbol{\Omega}-\boldsymbol{\Omega}_{e}\right)-\mathbf{J} \boldsymbol{\Omega} \times \boldsymbol{\Omega}_{e}+\mathrm{f}_{0}\left(\boldsymbol{\Omega}-\boldsymbol{\Omega}_{e}\right)\right\}
$$

where the term in braces represents the external inpurt stabilizing $\boldsymbol{\Omega}_{e}$. Thus,

$$
\mathbf{U}=\mathbf{f}(\boldsymbol{\Omega} ; \mathbf{J})=\mathbf{J} \boldsymbol{\Omega}_{e} \times\left(\boldsymbol{\Omega}-\boldsymbol{\Omega}_{e}\right)-\mathbf{J} \boldsymbol{\Omega} \times \boldsymbol{\Omega}_{e}+\mathbf{f}_{0}\left(\boldsymbol{\Omega}-\boldsymbol{\Omega}_{e}\right)
$$

However, in general we do not have exact knowledge of $\mathbf{J}$ so that the feedback will be designed on the basis of a nominal value $\mathbf{J}_{0}$ of the inertia, i.e., $\mathbf{U}=\mathbf{f}\left(\boldsymbol{\Omega} ; \mathbf{J}_{0}\right)$.

The closed loop system then becomes

$$
\mathbf{J} \dot{\boldsymbol{\Omega}}=\mathbf{J} \boldsymbol{\Omega} \times \boldsymbol{\Omega}+\left\{\mathbf{J}_{0} \boldsymbol{\Omega}_{e} \times\left(\boldsymbol{\Omega}-\boldsymbol{\Omega}_{e}\right)-\mathbf{J}_{0} \boldsymbol{\Omega} \times \boldsymbol{\Omega}_{e}+\mathbf{f}_{0}\left(\boldsymbol{\Omega}-\boldsymbol{\Omega}_{e}\right)\right\}
$$

In general, $\boldsymbol{\Omega}_{e}$ will no longer be an equilibrium of the closed loop system. Therefore, the control law cannot robustly stabilizes $\boldsymbol{\Omega}_{e}$ with respect to the parametric uncertainty in $\mathbf{J}$. As a concrete example, consider a given angular velocity $\boldsymbol{\Omega}_{e}=(3.0,6.0,2.0)^{T}$ that we seek to stabilize. Assume a nominal inertia matrix $\mathbf{J}_{0}=\operatorname{diag}(4.0,6.0,8.0)$ and an actual inertia matrix $\mathbf{J}=\operatorname{diag}(4.2,6.1,7.9)$. In [2] a control law for the stabilization of the null solution of Euler's equation is given. For $\mathbf{J}=\operatorname{diag}\left(j_{1}, j_{2}, j_{3}\right)$ and $\boldsymbol{\Omega}=\left(\omega_{1}, \omega_{2}, \omega_{3}\right)^{T}$, it has the form $\mathbf{U}^{*}=\left(0,0, v_{3}\right)^{T}$, where $v_{3}=-\left(j_{1}-\right.$ 
$\left.j_{2}\right) \omega_{1} \omega_{2}+j_{3}\left(k \omega_{1}+n \omega_{2}+m \omega_{3}+F\left(\omega_{1}, \omega_{2}, \omega_{3}\right)\right), k, n$ are real numbers, $m \leq 0$ and $F$ contains terms of order two and/or higher. Choosing $k=n=1, m=-1, F \equiv 0$ and transforming as suggested above, one gets $\mathbf{U}=f(\boldsymbol{\Omega})=\left(u_{1}, u_{2}, u_{3}\right)^{T}$ for stabilizing $\boldsymbol{\Omega}_{e}=(3.0,6.0,2.0)^{T}$ based on the nominal inertia matrix $\mathbf{J}_{0}$, where

$$
\left.\begin{array}{l}
u_{1}=-28 \omega_{2}+84 \omega_{3}+24, \\
u_{2}=24 \omega_{1}-36 \omega_{3}-24, \\
u_{3}=-64 \omega_{1}+32 \omega_{2}-8 \omega_{3}+2 \omega_{1} \omega_{2}+16 .
\end{array}\right\}
$$

This control law needs three torques to stabilize zero angular velocity, although the original (untransformed) control law needs only one torque.

On the other hand, by Corollary 3.1 , a control law based on $\mathbf{J}_{0}$, is as follows $(\lambda=50)$ :

$$
\begin{aligned}
& u_{1}=-84 \omega_{2}+264 \omega_{3}, \\
& u_{2}=84 \omega_{1}-138 \omega_{3}, \\
& u_{3}=-264 \omega_{1}+138 \omega_{2} .
\end{aligned}
$$

Figure 4 compares the control law given in (6.2) and the one computed here. The curves represent angular velocities $\omega_{i}(t), i=1,2,3$, of the closed loop systems with initial angular velocity $\Omega_{0}=(3.01,6.01,2.01)^{T}$. The figure shows that the control law in (6.2) fails while the control law in (6.3) continues to work. This suggests that the family of control laws in Corollary 3.1 is robust to parametric uncertainty in $\mathbf{J}$.

We now analyze the system with parametric uncertainty. In what follows, $\|\mathbf{x}\|$ denotes the 2-norm of a vector $\mathbf{x},\|\mathbf{A}\|$ is the corresponding induced matrix norm of matrix $\mathbf{A}$. From Corollary 3.1, a feedback control law based on the nominal value $\mathbf{J}_{0}$ of $\mathbf{J}$ is $\mathbf{U}=\boldsymbol{\Omega} \times \mathbf{b}_{0}=\boldsymbol{\Omega} \times\left(\mathbf{J}_{0}-\lambda \mathbf{1}_{3}\right) \boldsymbol{\Omega}_{e}$, where $\lambda>\bar{\sigma}\left(\mathbf{J}_{0}\right)$ or $\lambda<\underline{\sigma}\left(\mathbf{J}_{0}\right)$. The closed loop system with this control law is

$$
\mathbf{J} \dot{\boldsymbol{\Omega}}=\left(\mathbf{J} \boldsymbol{\Omega}-\mathbf{b}_{0}\right) \times \boldsymbol{\Omega}=\left(\mathbf{J} \boldsymbol{\Omega}-\left(\mathbf{J}_{0}-\lambda \mathbf{1}_{3}\right) \boldsymbol{\Omega}_{e} \times \boldsymbol{\Omega}\right.
$$


Note that $\Omega_{e}$ is no longer an equilibrium point of (6.4). However, one still can judge whether a feedback control law is 'good' by examining the magnitude of $\left\|\boldsymbol{\Omega}(t)-\boldsymbol{\Omega}_{e}\right\|$. Moreover, we obtain a family of control laws depending on the paramter $\lambda$ rather than a single one. Thus, we introduce the following definition.

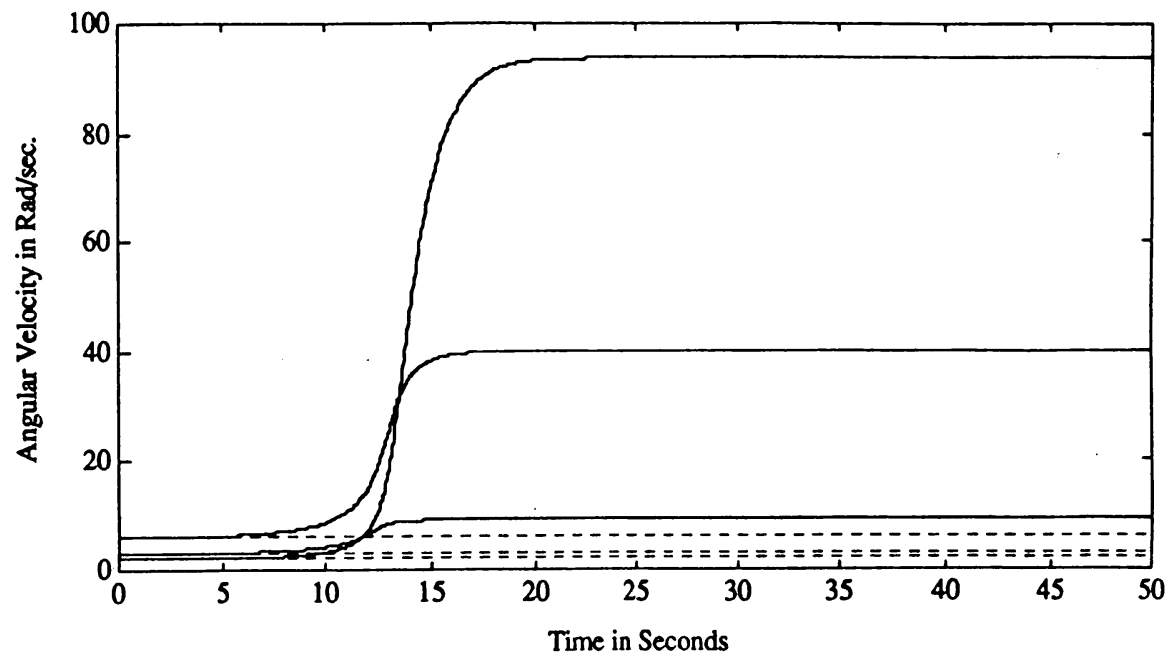

Figure 4. Comparison of two closed loop responses.

Definition 6.1. For an open loop system, $\dot{\mathbf{x}}=f(\mathbf{x} ; \mathbf{J}, \mathbf{U})$ depending on an uncertain matrix $\mathbf{J} \in Q=\left\{\mathbf{J}_{0}+\Delta \mathbf{J}:\|\Delta \mathbf{J}\| \leq \gamma\right\}$, and a family of feedback control laws $\mathbf{U}=g\left(\mathbf{x} ; \mathbf{J}_{0}, \lambda\right)$ is said to robustly stabilize a point $\mathbf{x}_{e}$ over $Q$ if for every given $\varepsilon>0$ there exists $\lambda_{0}$ and $\delta$, both independent of $\Delta \mathbf{J}$, such that $\left\|\mathbf{x}(t ; \mathbf{J}, \lambda)-\mathbf{x}_{e}\right\|<\varepsilon$ for all $t>0$ and for all $\mathbf{J} \in Q$, as $\left\|\mathbf{x}(0 ; \mathbf{J}, \lambda)-\mathbf{x}_{e}\right\|<\delta$ and $|\lambda| \geq \lambda_{0}$, where $\mathbf{x}(t ; \mathbf{J}, \lambda)$ is the solution of closed loop system $\dot{\mathbf{x}}=f\left(\mathbf{x} ; \mathbf{J}, \mathbf{g}\left(\mathbf{x} ; \mathbf{J}_{0}, \lambda\right)\right)$ with initial value $\mathbf{x}(0 ; \mathbf{J}, \lambda)$.

The robust stability of this definition refers to a family of control laws rather than a single one. Thus, in order to keep the trajectory close to the equilibrium, we need not only to choose the initial value close enough to the equilibrium, but also must choose the paramter $\lambda$ having large enough absolute value. We can now show that the family in Corollary 3.1 is robust in the sense of 


\section{Definition 6.1.}

Theorem 6.1. Let $Q=\left\{\mathbf{J}_{0}+\Delta \mathbf{J}:\|\Delta \mathbf{J}\| \leq \gamma\right\}$. Then, for the system (3.1), the family of feedback control laws defined in Corollary 3.1 robustly stabilize $\boldsymbol{\Omega}_{e}$ over $Q$.

Proof. First, note that $\boldsymbol{\Omega}_{e}$ is no longer an equilibrium of (6.4). Rewrite (6.4) as $\mathbf{J} \dot{\boldsymbol{\Omega}}=(\mathbf{J} \boldsymbol{\Omega}-$ $\left.\mathbf{b}_{0}\right) \times \boldsymbol{\Omega}=\left(\mathbf{J} \boldsymbol{\Omega}-\left(\mathbf{J}-\lambda \mathbf{1}_{3}\right) \boldsymbol{\Omega}_{e}^{*}\right) \times \boldsymbol{\Omega}$, where $\boldsymbol{\Omega}_{e}^{*}=\left(\mathbf{J}-\lambda \mathbf{1}_{3}\right)^{-1}\left(\mathbf{J}_{0}-\lambda \mathbf{1}_{3}\right) \boldsymbol{\Omega}_{e}$. Thus, by Corollary 3.1, the control law stabilizes a new equilibrium $\Omega_{e}^{*}$, if $\lambda>\lambda_{\max }(\mathbf{J})$ or $\lambda<\lambda_{\min }(\mathbf{J})$.

Claim. We have $\Omega_{e}^{*} \rightarrow \Omega_{e}$, as $|\lambda| \rightarrow+\infty$.

Proof. Note that for $|\lambda| \geq\left\|\mathbf{J}_{0}\right\|+\gamma,\left(\mathbf{J}_{0}+\boldsymbol{\Delta} \mathbf{J}-\lambda \mathbf{1}_{3}\right)$ is invertible and $\left\|\boldsymbol{\Omega}_{e}-\boldsymbol{\Omega}_{e}^{*}\right\| \leq \|\left(\mathbf{J}_{0}+\boldsymbol{\Delta} \mathbf{J}-\right.$ $\left.\lambda \mathbf{1}_{3}\right)^{-1} \Delta \mathbf{J}\|\| \boldsymbol{\Omega}_{e}\|\leq \gamma\|\left(\mathbf{J}_{0}+\Delta \mathbf{J}-\lambda \mathbf{1}_{3}\right)^{-1}\|\| \boldsymbol{\Omega}_{e}\left\|=\frac{\gamma}{|\lambda|}\right\|\left(\mathbf{1}_{3}-\frac{\mathbf{J}_{0}+\Delta b J}{\lambda}\right)^{-1}\|\| \boldsymbol{\Omega}_{e} \|$. However, for $\lambda$ satisfying $|\lambda| \geq\left\|\mathbf{J}_{0}\right\|+\gamma$, we have

$$
\left\|\frac{\mathbf{J}_{0}+\Delta \mathbf{J}}{\lambda}\right\| \leq \frac{1}{|\lambda|}\left\|\mathbf{J}_{0}+\Delta \mathbf{J}\right\| \leq \frac{1}{|\lambda|}\left(\left\|\mathbf{J}_{0}\right\|+\gamma\right) \leq 1
$$

Thus, $\left\|\left(\mathbf{1}_{3}-\frac{\mathbf{J}_{0}+\Delta \mathbf{J}}{\lambda}\right)^{-1}\right\| \leq\left(1-\left\|\frac{\mathbf{J}_{0}+\Delta \mathbf{J}}{\lambda}\right\|\right)^{-1}$ (see [22]) for $|\lambda| \geq\left\|\mathbf{J}_{0}\right\|+\gamma$. Consequently, $\left\|\boldsymbol{\Omega}_{e}-\boldsymbol{\Omega}_{e}^{*}\right\| \leq \frac{\gamma}{|\lambda|}\left(1-\left\|\frac{\mathbf{J}_{0}+\Delta \mathbf{J}}{\lambda}\right\|\right)^{-1}\left\|\boldsymbol{\Omega}_{e}\right\| \leq \frac{\gamma}{|\lambda|}\left(1-\frac{1}{|\lambda|}\left(\left\|\mathbf{J}_{0}\right\|+\gamma\right)\right)^{-1}\left\|\boldsymbol{\Omega}_{e}\right\|=\beta(\lambda)\left\|\boldsymbol{\Omega}_{e}\right\| \rightarrow 0$, as $|\lambda| \rightarrow+\infty$, where $\beta(\lambda):=\frac{\gamma}{|\lambda|}\left(1-\frac{1}{|\lambda|}\left(\left\|\mathbf{J}_{0}\right\|+\gamma\right)\right)^{-1}$ and claim has been proven.

Since $\Omega_{e}^{*}=\Omega_{e}^{*}(\mathbf{J}, \lambda)$ is a Lyapunov stable equilbrium of $(6.4)$, for a given $\varepsilon>0$, there exists a $\delta=\delta(\mathbf{J}, \lambda)$ determined by (6.1) such that $\left\|\boldsymbol{\Omega}(t ; \mathbf{J}, \lambda)-\boldsymbol{\Omega}_{e}^{*}\right\|<0.5 \varepsilon$, for all $t>0$, as $\left\|\boldsymbol{\Omega}(0 ; \mathbf{J}, \lambda)-\Omega_{e}^{*}(\mathbf{J}, \lambda)\right\|<\delta(\mathbf{J}, \lambda)$. Denote $\delta_{\text {inf }}(\lambda)=\inf \{\delta(\mathbf{J}, \lambda), \mathbf{J} \in Q\}$. By Corollary $6.2, \delta(\mathbf{J}, \lambda)$ is a continuous function of $\mathbf{J}$ over the closed set $Q$ for fixed $\lambda$. Thus, $\delta_{\text {inf }}(\lambda)$ is reached at some $\mathbf{J}^{*} \in Q$. Consequently, $\delta_{\text {inf }}(\lambda)=\delta\left(\mathbf{J}^{*}, \lambda\right)>0$. By Corollary $6.2, \delta(\mathbf{J}, \lambda)$ is a strictly increasing function of $|\lambda|$ for $\lambda \in\left(j_{1},+\infty\right)$ and $\lambda \in(-\infty, 0)$. Thus, $\delta_{\text {inf }}(\lambda)$ is non-decreasing function 
of $|\lambda|$ for $\lambda \in\left(j_{1},+\infty\right)$ and $\lambda \in(-\infty, 0)$. Moreover, $\delta_{\text {inf }}(\lambda)$ has an upper bound, $0.5 \varepsilon$, therefore $\delta_{\text {inf }}(\lambda) \rightarrow a<0.5 \varepsilon$, where $a>0$ as $|\lambda| \rightarrow+\infty$. On the other hand, $\beta(\lambda)\left\|\boldsymbol{\Omega}_{e}\right\| \rightarrow 0$ as $|\lambda| \rightarrow+\infty$. Thus there exists a positive number $\lambda_{0}$, such that $\beta(\lambda)\left\|\Omega_{e}\right\|<\delta_{\text {inf }}(\lambda)$ as $|\lambda| \geq \lambda_{0}$. Let $\delta_{1}=\delta_{\text {inf }}(\lambda)-\beta\left(\lambda_{0}\right)\left\|\Omega_{e}\right\|$. Note that $\lambda_{0}$ can be chosen greater than $\max \left\{\left|j_{1}\right|,\left|j_{3}\right|\right\}$, so that for any $\lambda$ satisfying $|\lambda| \geq \lambda_{0}, \delta_{1}<\delta_{\text {inf }}(\lambda)-\beta(\lambda)\left\|\boldsymbol{\Omega}_{e}\right\|<\delta_{\text {inf }}(\lambda)-\left\|\boldsymbol{\Omega}_{e}-\boldsymbol{\Omega}_{e}^{*}\right\|$. Here we used the fact that $\delta_{\text {inf }}(\lambda)$ is non-decreasing and $\beta(\lambda)\left\|\boldsymbol{\Omega}_{e}\right\|$ approaches zero. Thus, for any $\lambda$ satisfying $|\lambda| \geq \lambda_{0},\left\|\boldsymbol{\Omega}(0 ; \mathbf{J}, \lambda)-\boldsymbol{\Omega}_{e}\right\|<\delta_{1}$ implies $\left\|\boldsymbol{\Omega}(0 ; \mathbf{J}, \lambda)-\boldsymbol{\Omega}_{e}^{*}\left(\lambda_{0}, \mathbf{J}, \lambda\right)\right\|<\delta_{\text {inf }}(\lambda) \Rightarrow$ $\left\|\boldsymbol{\Omega}(t ; \mathbf{J}, \lambda)-\boldsymbol{\Omega}_{e}^{*}\left(\lambda_{0}, \mathbf{J}, \lambda\right)\right\|<0.5 \varepsilon$. Finally, since $\left\|\boldsymbol{\Omega}_{e}^{*}\left(\lambda_{0}, \mathbf{J}, \lambda\right)-\boldsymbol{\Omega}_{e}\right\| \leq \beta(\lambda)\left\|\boldsymbol{\Omega}_{e}\right\|<\delta_{\text {inf }}(\lambda)<0.5 \varepsilon$ we have $\left\|\boldsymbol{\Omega}(t ; J, \lambda)-\boldsymbol{\Omega}_{e}\right\| \leq\left\|\boldsymbol{\Omega}(t)-\boldsymbol{\Omega}_{e}^{*}\right\|+\| \boldsymbol{\Omega}_{e}^{*}-\boldsymbol{\Omega}_{e} \mid \leq 0.5 \varepsilon+0.5 \varepsilon=\varepsilon$ for all $t \geq 0$, all $\mathbf{J} \in Q$, $\left\|\boldsymbol{\Omega}(0 ; \mathbf{J}, \lambda)-\boldsymbol{\Omega}_{e}\right\|<\delta_{1}$ and $|\lambda| \geq \lambda_{0}$.

Remark. The control laws in Corollaries 4.1 - 4.2 are not robust. Thus, to use these control laws, an accurate measurement of $\mathbf{J}$ is essential. However, it is easy to estimate the error in the rotation axis caused by the error in $\mathbf{J}$. For example, suppose the nominal inertia matrix $\mathbf{J}_{0}=\operatorname{diag}\left(j_{01}, j_{02}, j_{03}\right)$, $\mathbf{J}=\mathbf{J}_{0}+\boldsymbol{\Delta} \mathbf{J}, \boldsymbol{\Delta} \mathbf{J}=\operatorname{diag}\left(\Delta j_{1}, \Delta j_{2}, \Delta j_{3}\right)$ and $\|\boldsymbol{\Delta} J\| \leq \gamma$. Corollary 4.2 then gives $\mathbf{U}=\left(0, u_{2}, 0\right)^{T}$, where

$$
\begin{aligned}
u_{2} & =\left(j_{01}-j_{02}\right) w_{3} \omega_{1}+\left(j_{02}-j_{03}\right) w_{1} \omega_{3} \\
& =\left(j_{1}-j_{2}\right) \frac{j_{01}-j_{02}}{j_{1}-j_{2}} w_{3} \omega_{1}+\left(j_{2}-j_{3}\right) \frac{j_{02}-j_{03}}{j_{2}-j_{3}} w_{1} \omega_{3}, \\
& =\left(j_{1}-j_{2}\right) w_{3}^{*} \omega_{1}+\left(j_{2}-j_{3}\right) w_{1}^{*} \omega_{3}
\end{aligned}
$$

and $w_{3}^{*}=\frac{j_{01}-j_{02}}{j_{1}-j_{2}} w_{3}, w_{1}^{*}=\frac{j_{02}-j_{03}}{j_{2}-j_{3}} w_{1}$.

Thus, the control law stabilizes $\boldsymbol{\Omega}_{e}^{*}=\left(w_{1}^{*}, 0, w_{3}^{*}\right)^{T}$, which is close to $\boldsymbol{\Omega}_{e}$. An estimate of the relative error in the rotation axis can be computed as follows:

$$
\left\|\boldsymbol{\Omega}_{e}^{*}-\boldsymbol{\Omega}_{e}\right\|=\left\{\left(w_{1}^{*}-w_{1}\right)^{2}+\left(w_{3}^{*}-w_{3}\right)^{2}\right\}^{\frac{1}{2}}
$$




$$
\begin{aligned}
& \leq\left\{\left(\frac{\Delta j_{1}-\Delta j_{2}}{j_{1}-j_{2}}\right)^{2} w_{1}^{2}+\left(\frac{\Delta j_{2}-\Delta j_{3}}{j_{2}-j_{3}}\right)^{2} w_{3}^{2}\right\}^{\frac{1}{2}} \\
& \leq \frac{2 \gamma}{M}\left\|\boldsymbol{\Omega}_{e}\right\|,
\end{aligned}
$$

where $M=\max \left\{\left|j_{01}-j_{02}\right|-2 \gamma,\left|j_{02}-j_{03}\right|-2 \gamma\right\}$. Thus, $\frac{\left\|\boldsymbol{\Omega}_{e}^{*}-\boldsymbol{\Omega}_{e}\right\|}{\left\|\boldsymbol{\Omega}_{e}\right\|} \leq \frac{2 \gamma}{M}$. For small $\gamma$, the relative error is small.

\section{$\S 7$. Conclusion}

In this paper, we have generalized the stabilization problem of a uniformly-rotating rigid body by considering the stabilizion of an arbitrary angular velocity. As we have shown in $\S 6.2$, this problem is not equivalent to the problem of stabilizing a zero angular velocity if the robustness of the proposed control laws is a consideration. In this paper, we have presented a family of linear control laws which stabilize an arbitrary angular velocity and proved them robust with respect to parmetric uncertainty in the inertia values of the sense of Definition 6.1. In some important cases (including stabilizing the intermediate princpal axis), we have presented stabilizing control laws that use only one applied torque aligned with a coordinate axis. These one-torque control laws are "almost" robust: A small error in the inertia values causes only a small, relative error in the axis of rotation. We gave an estimate of the relative error. This work was done in a general coordinate frame rather than a princpal-axes one. The principal-axes frame may not be convenient in practical applications. The methodology we have used here is based on the energy-momentum method, which is a powerful method in the stability analysis of motions including both rotational and deformational modes. In a subsequent paper we shall extend the method here to design robustly stabilizing control laws for more complex systems, such as those incorporating a flexible element modeled as a geometric exact rod, and investigate the possibility of reducing the number of applied control torques. We also plan to investigate the control of bifurcations that may occur in rotating systems by the methodology employed in this paper. 


\section{Acknowledgement}

The author's would like to thank Eyad Abed, Tony Bloch, P.S. Krishnaprasad and Jerry Marsden for their suggestions and comments on this work.

\section{References}

[1] R. Abraham and J.E. Marsden, Foundations of Mechanics, Second Edition, Addison-Wesley, Reading, 1978.

[2] D. Aeyels, Stabilization by smooth feedback of the angular velocity of a rigid body, Systems \& Control Letters, 5, 59-63, 1985.

[3] D. Aeyels and M. Szafranski, Comments on the stabilization of the angular velocity of a rigid body, Systems \& Control Letters, 10, 35-39, 1985.

[4] V.I. Arnold, Mathematical Methods of Classical Mechanics: Graduate Texts in Math, 60, Sringer-Verlag, New York, 1978.

[5] V.I. Arnold (ed.), Dynamical Systems III, EMS, Vol. 3, Springer-Verlag, New York, 1988.

[6] A. M. Bloch and J. E. Marsden, Stabilization of rigid body dynamics by the energy-Casimir method, System \& Control Letters, 14, 341-346, 1990.

[7] A. M. Bloch, P. S. Krishnaprasad, J. E. Marsden and G. Sánchez de Alverez, Stabilization of rigid body dynamics by internal and external torques, Automatica, 28, 745-756, 1992.

[8] R. W. Brockett, Asymptotic stability and feedback stabilization, found in Differential Geometric Control Theory, Progress in Mathematics, Vol. 27, R. W. Brockett et. al, eds., 181-191, Birkhauser, Boston, 1983.

[9] C. I. Byrnes and A. Isidori, Attitude stabilization of rigid spacecraft, Automatica, 27:1, 87-96, 1990. 
[10] P. E. Crouch, Spacecraft attitude control and stabilization: Application of geometric control to rigid body models, IEEE Trans. on Automatic Contr., AC-29, 321-331, 1986.

[11] A. Isidori, Nonlinear Control Systems, Second Edition, Springer-Verlag, Berlin, 1989.

[12] P. S. Krishnaprasad, Lie - Poisson Structures, Dual - Spin Spacecraft and Asymptotic Stability, Nonlinear Analysis, Theory, Methods and Applications, 10, 1011-1035, 1985.

[13] P. S. Krishnaprasad and J. E. Marsden, Hamiltonian structure and stability for rigid bodies with flexible attachments, Arch. Rational Mech. Anal., 98, 71-93, 1987.

[14] J. E. Marsden and A. Weinstein, Reduction of symplectic manifold with symmetry, Rep. Math. Phys., 5, 121-130, 1974.

[15] H. Nijmeijer and A. J. van der Schaft, Nonlinear Dynamical Control Systems, Springer-Verlag, New York, 1990.

[16] R. Outbib and G. Sallet, Stabilizability of the angular velocity of a rigid body revisited, Systems and Control Letters, 18, 93-98, 1992.

[17] L. S. Pontryagin, Ordinary differential equations, Addison-Wesley, Reading, 1962.

[18] T. A. Posbergh and R. Zhao, Stabilization of the uniform rotation of a rigid body by the energy-momentum method, Fields Institute Communications, 1, 263-280, 1993.

[19] F. Scheck, Mechanics, Springer-Verlag, New York, 1990.

[20] C. I. Siegel and J. K. Moser, Lectures on Celestial Mechanics, Springer-Verlag, New York, 1971.

[21] J. C. Simo, T. A. Posbergh and J. E. Marsden, Stability of coupled rigid body and geometrically exact rods: block diagonalization and the energy-momentum method, Physics Reports, 193, 280-360, 1991.

[22] J. C. Simo, D. R. Lewis and J. E. Marsden, Stability of relative equilibria I: The reduced energy-momentum method, Arch. Rat. Mech. Anal., 115, 15-59, 1990. 
[23] J. C. Simo, T. A. Posbergh and J. E. Marsden, Stability of relative equilibria II: Three dimensional elasticity, Arch. Rat. Mech. Anal., 115, 61-100, 1991.

[24] L. Taylor Jr. and A. V. Balakrishnan, A mathematical problem and a spacecraft control laboratory experiment (SCOLE) used to evaluate control laws for flexible spacecraft, $N A S A$ Report, 1984.

[25] C.-J. Wan and D.S. Berstein, Rotational stabilization of a rigid body using two torque actuators, to appear in Proceedings of the 32nd Conference on Decision and Control, 1993.

[26] L. S. Wang, Geometry, Dynamics and control of coupled systems, Ph.D. Dissertation, Department of Electrical Engineering, University of Maryland, 1990.

[27] L.S. Wang and P.S. Krishnaprasad, Gyroscopic control and stabilization, J. Nonlinear Sci., 2, 367-415, 1992.

[28] R. Zhao and T. A. Posbergh, Stabilization of a rotating rigid body by the energy-momentum method, Proceedings of 31st Conf. on Decision and Control, Tucson, 1583-1588, 1992.

[29] R. Zhao and T. A. Posbergh, Feedback stabilization of a uniform rigid body rotation, to appear in Systems \& Control Letters, 1993. 


\section{Appendix A. Hamiltonian systems}

For a conservative mechanical system with $n$-degrees of freedom, the motion satisfies the first order equations

$$
\dot{q}^{i}=\frac{\partial H}{\partial p_{i}}, \quad \dot{p}_{i}=-\frac{\partial H}{\partial q^{i}}, \quad i=1, \ldots, n .
$$

Here $H$ is the hamiltonian of the system, $q^{i}$ are the configuration variables and $p_{i}$ are the corresponding momentum variables. Equations (A.1) are called Hamiltonian's equations.

A more geometric description of such a hamiltonian system is that of a flow on a manifold. This can be obtained from (A.1) as follows. Let $\mathbf{x}=(\mathbf{q}, \mathbf{p})$ denote a vector of the configuration and momentum variables. Then (A.1) takes the form

$$
\dot{\mathbf{x}}-\mathbf{J} d H(\mathbf{x})
$$

where $d H(\mathbf{x})=\left(\frac{\partial H}{\partial q^{i}}, \frac{\partial H}{\partial p_{i}}\right)^{T}, i=1, \ldots, n$ is the gradient of $H$ and $\mathbf{J}=\left[\begin{array}{cc}0 & \mathbf{1}_{n} \\ -\mathbf{1}_{n} & 0\end{array}\right]\left(\mathbf{1}_{n}\right.$ is $n \times n$ identity matrix). The vector $\mathbf{J} d H(\mathbf{x})$ is called the hamiltonian vector field and is denoted by $\mathcal{X}_{H}$. Now define $\Omega(\mathbf{a}, \mathbf{b})=\mathbf{J}^{-1} \mathbf{a} \cdot \mathbf{b}$, where $\cdot$ denotes the standard dot product and clearly $\Omega\left(\mathcal{X}_{H}, \mathbf{b}\right)=$ $d H(\mathbf{x}) \cdot$ b. It is straghtforward to show that $\Omega(\cdot, \cdot)$ is skew symmetric and bilinear. In general, a skew-symmetric, bilinear form $\Omega(\cdot, \cdot)$ defined on $\mathbb{R}^{2 n} \times \mathbb{R}^{2 n}$ is called a sympletic form in $\mathbb{R}^{2 n}$. A hamiltonian vector field $\mathcal{X}_{H}$ is defined as a vector field satisfying $\Omega\left(\mathcal{X}_{H}, \mathbf{b}\right)=d H(\mathbf{x}) \cdot \mathbf{b}$. We summarize these ideas in the following definitions.

Definition 1.1. Let $M^{2 n}$ be a $2 n$-dimensional, differentiable manifold. Suppose there is a skewsymmectric, bilinear form $\Omega_{z}(\mathbf{a}, \mathbf{b})$ defined on each tangent space to $M^{2 n}$ at each point $z$ of $M^{2 n}$. If $\Omega_{z}(a, b)$ is non-degenerate, closed (cf. [1]) and differentiable with respect to $z$, then we say that a symplectic structure $\Omega$ is given on $M^{2 n}$ and call $\left(M^{2 n}, \Omega\right)$ a symplectic manifold.

A coordinate-free definition of a hamiltonian vector field is then given in the following. Here $P$ denotes the phase spare of the system corresponding to the set of all configurations and corresponding momenta.

Definition 1.2. Let $(P, \Omega)$ be a symplectic manifold. A vector field $\mathcal{X}$ on $P$ is called hamiltonian if there is a function $H: P \rightarrow \mathbb{R}$ such that

$$
\Omega_{z}(\mathcal{X}(z), Y)=d H(z) \cdot Y
$$

In this case we write $\mathcal{X}_{H}$ for $\mathcal{X}$. The triple $\left(P, \Omega, \mathcal{X}_{H}\right)$ is called a hamiltonian system. 
It can be shown that for any function $H$ on $P$, the hamiltonian vector field $\mathcal{X}_{H}$ exists and is unique (cf. [1]).

As in the classical theory, the hamiltonian $H$ is conserved under the flow (cf [1]).

\section{Appendix B. The proof of Proposition 6.1.}

We start from Euler's equations (3.2b), then apply the result to (3.2a).

Case $1 . J$ is a diagnonal matrix, i.e., $J=\operatorname{diag}\left(j_{1}, j_{2}, j_{3}\right)$. For a given $\varepsilon^{*}$, there is a corresponding $\delta^{*}$ defined in the proof of Theorem 6.1. We want to compute $\delta^{*}$ in terms of $\varepsilon^{*}$, the eigenvalues of $J$ and $\lambda$. After some algebraic computation, we get $H^{*}(\boldsymbol{\Pi})=H^{\mu}(\boldsymbol{\Pi})-\frac{\lambda}{2}\left(\|\boldsymbol{\Pi}\|^{2}-\left\|\boldsymbol{\Pi}_{e}\right\|^{2}\right)=$ $\frac{1}{2}\left(\boldsymbol{\Pi}-\boldsymbol{\Pi}_{e}\right) \cdot \mathbf{J}(\lambda)^{-1}\left(\boldsymbol{\Pi}-\boldsymbol{\Pi}_{e}\right)+C(\lambda)$, where $\mathbf{J}(\lambda)=\operatorname{diag}\left(j_{1} \lambda\left(\lambda-j_{1}\right)^{-1}, j_{2} \lambda\left(\lambda-j_{2}\right)^{-1}, j_{3} \lambda\left(\lambda-j_{3}\right)^{-1}\right)$, and $C(\lambda)=\frac{1}{2 \lambda^{2}} \Pi_{e} \cdot J \Pi_{e}$.

Subcase $1^{\circ}: \lambda>j_{1}$.

Claim. $h_{\min }\left(\varepsilon^{*}\right)=\frac{1}{2}\left(\frac{\varepsilon^{*^{2}}}{j_{1} \lambda\left(\lambda-j_{1}\right)^{-1}}\right)+C(\lambda)$.

Proof. Define $H_{\min }(\boldsymbol{\Pi}):=\frac{1}{2 c_{\max }}\left\|\boldsymbol{\Pi}-\boldsymbol{\Pi}_{e}\right\|^{2}+C(\lambda)$, where $c_{\max }=j_{1} \lambda\left(\lambda-j_{1}\right)^{-1}$. Then, $H_{\min }(\Pi)=\frac{1}{2}\left(\frac{\varepsilon^{*^{2}}}{j_{1} \lambda\left(\lambda-j_{1}\right)^{-1}}\right)+C(\lambda)$ for all $\Pi \in \partial B\left(\Pi_{e}, \varepsilon^{*}\right)$. Since $c_{\max }=j_{1} \lambda\left(\lambda-j_{1}\right)^{-1}>$ $j_{2} \lambda\left(\lambda-j_{2}\right)^{-1}>j_{3} \lambda\left(\lambda-j_{3}\right)^{-1}$, one sees that $H^{*}(\Pi) \geq H_{\min }(\Pi)=\frac{1}{2}\left(\frac{\varepsilon^{*^{2}}}{j_{1} \lambda\left(\lambda-j_{1}\right)^{-1}}\right)+C(\lambda)$, for all $\boldsymbol{\Pi} \in \partial B\left(\boldsymbol{\Pi}_{e}, \varepsilon^{*}\right)$. On the other hand, for $\boldsymbol{\Pi}^{*}=\boldsymbol{\Pi}_{e}+\left(\varepsilon^{*}, 0,0\right)^{T} \in \partial B\left(\boldsymbol{\Pi}_{e}, \varepsilon^{*}\right), H^{*}\left(\boldsymbol{\Pi}^{*}\right)=$ $\frac{1}{2}\left(\frac{\varepsilon^{*^{2}}}{j_{1} \lambda\left(\lambda-j_{1}\right)^{-1}}\right)+C(\lambda)$. Thus, by definition of $h_{\min }$ (see the proof of Theorem 6.1$), h_{\min }\left(\varepsilon^{*}\right)=$ $\frac{1}{2}\left(\frac{\varepsilon^{*^{2}}}{j_{1} \lambda\left(\lambda-j_{1}\right)^{-1}}\right)+C(\lambda)$. Now define $H_{\max }(\boldsymbol{\Pi}):=\frac{1}{2 c_{\min }}\left\|\boldsymbol{\Pi}-\Pi_{e}\right\|^{2}+C(\lambda)$, where $c_{\min }=$ $j_{3} \lambda\left(\lambda-j_{3}\right)^{-1}$. Then $H_{\max }(\boldsymbol{\Pi})=M(a)=\frac{1}{2}\left(\frac{a^{2}}{j_{3} \lambda\left(\lambda-j_{3}\right)^{-1}}\right)+C(\lambda)$ for all $\boldsymbol{\Pi} \in \partial B\left(\boldsymbol{\Pi}_{e}, a\right)$. Thus, $H^{*}(\boldsymbol{\Pi}) \leq M(a)$ for all $\Pi \in \partial B\left(\boldsymbol{\Pi}_{e}, a\right)$. Let $M(a)=h_{\min }\left(\varepsilon^{*}\right)$, i.e., $\frac{a^{2}}{j_{3} \lambda\left(\lambda-j_{3}\right)^{-1}}=\frac{\varepsilon^{*^{2}}}{j_{1} \lambda\left(\lambda-j_{1}\right)^{-1}}$ one gets $a=\left(\frac{j_{3}\left(\lambda-j_{1}\right)}{j_{1}\left(\lambda-j_{3}\right)}\right)^{\frac{1}{2}} \varepsilon^{*}$. One checks that $a<\varepsilon^{*}$. We claim that $\delta^{*}=a$. In fact, for any $\boldsymbol{\Pi}_{0} \in B\left(\boldsymbol{\Pi}_{e}, a\right) \subset \bar{B}\left(\boldsymbol{\Pi}_{e}, \varepsilon^{*}\right)$, let $r=\left\|\boldsymbol{\Pi}_{e^{-}}-\boldsymbol{\Pi}_{0}\right\|$, then $\boldsymbol{\Pi}_{0} \in \partial B\left(\boldsymbol{\Pi}_{e}, r\right)$ and $M(r)=$ 
$\frac{1}{2}\left(\frac{r^{2}}{j_{3} \lambda\left(\lambda-j_{3}\right)^{-1}}\right)+C(\lambda)<\frac{1}{2}\left(\frac{a^{2}}{j_{3} \lambda\left(\lambda-j_{3}\right)^{-1}}\right)+C(\lambda)=\frac{1}{2}\left(\frac{\varepsilon^{*^{2}}}{j_{1} \lambda\left(\lambda-j_{1}\right)^{-1}}\right)+C(\lambda)=h_{\min }\left(\varepsilon^{*}\right)$.

Thus $H^{*}\left(\boldsymbol{\Pi}_{0}\right) \leq M(r)<h_{\min }\left(\varepsilon^{*}\right)$. Hence, $\boldsymbol{\Pi}_{0} \in F\left(\varepsilon^{*^{*}}\right)$. Consequently, $B\left(\boldsymbol{\Pi}_{e}, a\right) \subset F\left(\varepsilon^{*}\right)$. On the other hand, for $R \in\left(a, \varepsilon^{*}\right)$, consider $\Pi^{* *}=\Pi_{e}+\left(0,0, \frac{R+a}{2}\right)^{T}$, then $\Pi^{* *} \in B\left(\Pi_{e}, \mathbb{P}\right) \subset \bar{B}\left(\Pi_{e}, \varepsilon^{*}\right)$ and $H^{*}\left(\boldsymbol{\Pi}^{* *}\right)=\frac{1}{2}\left(\frac{\left(\frac{R+a}{2}\right)^{2}}{j_{3} \lambda\left(\lambda-j_{3}\right)^{-1}}\right)+C(\lambda) \geq \frac{1}{2}\left(\frac{a^{2}}{j_{3} \lambda\left(\lambda-j_{3}\right)^{-1}}\right)+C(\lambda)=\frac{1}{2}\left(\frac{\varepsilon^{*^{2}}}{j_{1} \lambda\left(\lambda-j_{1}\right)^{-1}}\right)$ $+C(\lambda)=h_{\min }\left(\varepsilon^{*}\right)$. That is, $B\left(\boldsymbol{\Pi}_{e}, \mathbb{R}\right) \not \subset F\left(\varepsilon^{*}\right)$. By definition, $\delta^{*}=a=\left(\frac{j_{3}\left(\lambda-j_{1}\right)}{j_{1}\left(\lambda-j_{3}\right)} P\right)^{\frac{1}{2}} \varepsilon^{*}$.

For (3.3), substituting $\varepsilon^{*}=\varepsilon j_{1}^{-1}$ and $\delta$, one gets $\delta=\frac{j_{3}}{j_{1}}\left(\frac{j_{3}\left(\lambda-j_{1}\right)}{j_{1}\left(\lambda-j_{3}\right)}\right)^{\frac{1}{2}} \varepsilon$.

Subcase $2^{\circ} . \lambda<j_{3}$. The proof is similar to Subcase $1^{\circ}$.

Case 2. $\mathbf{J}$ is not a diagonal matrix. Do a coordinate transformation and diagonalize $\mathbf{J}$ to $\mathbf{J}_{P}=$ $\mathbf{X}^{T} \mathbf{J X}$, where $\mathbf{J}_{P}=\operatorname{diag}\left(j_{1}, j_{2}, j_{3}\right), j_{1}>j_{2}>j_{3}$, and $\mathbf{X} \in S O(3)$. Thus, under the new coordinate system, the body angular velocity $\Omega_{P}(t)=\mathbf{X}^{T} \Omega(t)$. Euler's equations then become

$$
\mathbf{J}_{P} \dot{\Omega}_{P}=\left(\mathbf{J}_{P} \boldsymbol{\Omega}_{P}-\mathrm{b}_{0 P}\right) \times \Omega_{P}
$$

where $\mathbf{b}_{0 P}=\mathbf{X}^{T} \mathbf{b}_{0}=\mathbf{X}^{T}\left(\mathbf{J} \boldsymbol{\Omega}_{e}-\lambda \boldsymbol{\Omega}_{e}\right)=\mathbf{J}_{P} \boldsymbol{\Omega}_{P e}-\lambda \boldsymbol{\Omega}_{P e}, \boldsymbol{\Omega}_{P e}=\mathbf{X}^{T} \boldsymbol{\Omega}_{e}$.

For $\lambda>j_{1}$ or $\lambda<j_{3}, \Omega_{P e}$ is a stable equilibrium of (B.1). Thus, for a given $\varepsilon>0$, let $\delta=\frac{j_{3}}{j_{1}}\left(\frac{j_{3}\left(\lambda-j_{1}\right)}{j_{1}\left(\lambda-j_{3}\right)}\right)^{\frac{1}{2}} \varepsilon$ if $\lambda>j_{j} ; \frac{j_{3}}{j_{1}}\left(\frac{j_{1}\left(j_{3}-\lambda\right)}{j_{3}\left(j_{1}-\lambda\right)}\right)^{\frac{1}{2}} \varepsilon$, if $\lambda>j_{3}$.

Then from $\underline{\text { Case } 1},\left\|\boldsymbol{\Omega}_{P}(t)-\boldsymbol{\Omega}_{P e}\right\|=\left\|\boldsymbol{\Omega}(t)-\boldsymbol{\Omega}_{e}\right\|<\varepsilon$, as $\left\|\boldsymbol{\Omega}_{P}(0)-\boldsymbol{\Omega}_{P e}\right\|=\left\|\boldsymbol{\Omega}(0)-\boldsymbol{\Omega}_{e}\right\|<\delta$. 
1108 Lawrence Markus, A brief history of control

Richard A. Brualdi, Keith L. Chavey \& Bryan L. Shader, Bipartite graphs and inverse sign patterns of strong sign-nonsingular matrices

A. Kersch, W. Morokoff \& A. Schuster, Radiative heat transfer with quasi-monte carlo methods

Jianhua Zhang, A free boundary problem arising from swelling-controlled release processes

Walter Littman \& Stephen Taylor, Local smoothing and energy decay for a semi-infinite beam pinned at several points and applications to boundary control

1113 Srdjan Stojanovic \& Thomas Svobodny, A free boundary problem for the Stokes equation via nonsmooth analysis

1114 Bronislaw Jakubczyk, Filtered differential algebras are complete invariants of static feedback

1115

1116

Boris Mordukhovich, Discrete approximations and refined Euler-Lagrange conditions for nonconvex differential inclusions

Bei Hu \& Hong-Ming Yin, The profile near blowup time for solution of the heat equation with a nonlinear boundary condition

Jin Ma \& Jiongmin Yong, Solvability of forward-backward SDEs and the nodal set of Hamilton-Jacobi-Bellman Equations

Chaocheng Huang \& Jiongmin Yong, Coupled parabolic and hyperbolic equations modeling age-dependent epidemic dynamics with nonlinear diffusion

Jiongmin Yong, Necessary conditions for minimax control problems of second order elliptic partial differential equations

Eitan Altman \& Nahum Shimkin, Worst-case and Nash routing policies in parallel queues with uncertain service allocations

Nahum Shimkin \& Adam Shwartz, Asymptotically efficient adaptive strategies in repeated games, part II: Asymptotic optimality

M.E. Bradley, Well-posedness and regularity results for a dynamic Von Kármán plate

Zhangxin Chen, Finite element analysis of the 1D full drift diffusion semiconductor model

Gang Bao \& David C. Dobson, Diffractive optics in nonlinear media with periodic structure

Steven Cox \& Enrique Zuazua, The rate at which energy decays in a damped string

Anthony W. Leung, Optimal control for nonlinear systems of partial differential equations related to ecology

H.J. Sussmann, A continuation method for nonholonomic path-finding problems

Yung-Jen Guo \& Walter Littman, The null boundary ,controllability for semilinear heat equations

Q. Zhang \& G. Yin, Turnpike sets in stochastic manufacturing systems with finite time horizon

I. Györi, F. Hartung \& J. Turi, Approximation of functional differential equations with time- and state-dependent delays by equations with piecewise constant arguments

I. Györi, F. Hartung \& J. Turi, Stability in delay equations with perturbed time lags

F. Hartung \& J. Turi, On the asymptotic behavior of the solutions of a state-dependent delay equation

Pierre-Alain Gremaud, Numerical optimization and quasiconvexity

Jie Tai Yu, Resultants and inversion formula for $N$ polynomials in $N$ variables

Avner Friedman \& J.L. Velázquez, The analysis of coating flows in a strip

Eduardo D. Sontag, Control of systems without drift via generic loops

Yuan Wang \& Eduardo D. Sontag, Orders of input/output differential equations and state space dimensions

Scott W. Hansen, Boundary control of a one-dimensional, linear, thermoelastic rod

Robert Lipton \& Bogdan Vernescu, Homogenization of two phase emulsions with surface tension effects

Scott Hansen \& Enrique Zuazua, Exact controllability and stabilization of a vibrating string with an interior point mass

1141 Bei Hu \& Jiongmin Yong, Pontryagin Maximum principle for semilinear and quasilinear parabolic equations with pointwise state constraints

Mark H.A. Davis, A deterministic approach to optimal stopping with application to a prophet inequality

M.H.A. Davis \& M. Zervos, A problem of singular stochastic control with discretionary stopping

Bernardo Cockburn \& Pierre-Alain Gremaud, An error estimate for finite element methods for scalar conservation laws

David C. Dobson \& Fadil Santosa, An image enhancement technique for electrical impedance tomography

Jin Ma, Philip Protter, \& Jiongmin Yong, Solving forward-backward stochastic differential equations explicitly - a four step scheme

Yong Liu, The equilibrium plasma subject to skin effect

Ulrich Hornung, Models for flow and transport through porous media derived by homogenization

Avner Friedman, Chaocheng Huang, \& Jiongmin Yong, Effective permeability of the boundary of a domain

Gang Bao, A uniqueness theorem for an inverse problem in periodic diffractive optics

Angelo Favini, Mary Ann Horn,\& Irena Lasiecka, Global existence and uniqueness of regular solutions to the dynamic von Kármán system with nonlinear boundary dissipation 
E.G. Kalnins \& Willard Miller, Jr., Models of $q$-algebra representations: $q$-integral transforms and "addition theorems"

E.G. Kalnins, V.B. Kuznetsov \& Willard Miller, Jr., Quadrics on complex Riemannian spaces of constant curvature, separation of variables and the Gaudin magnet

A. Kersch, W. Morokoff \& Chr. Werner, Selfconsistent simulation of sputtering with the DSMC method

Gang Bao, Finite element approximation of time harmonic waves in periodic structures

Tao Lin \& Hong Wang, Recovering the gradients of the solutions of second-order hyperbolic equations by interpolating the finite element solutions

Zhangxin Chen, $L^{p}$-posteriori error analysis of mixed methods for linear and quasilinear elliptic problems

Todd Arbogast \& Zhangxin Chen, Homogenization of compositional flow in fractured porous media

L. Qiu, B. Bernhardsson, A. Rantzer, E.J. Davison, P.M. Young \& J.C. Doyle, A formula for computation of the real stability radius

Maria Inés Troparevsky, Adaptive control of linear discrete time systems with external disturbances under inaccurate modelling: A case study

Petr Klouček \& Franz S. Rys, Stability of the fractional step $\Theta$-scheme for the nonstationary Navier-Stokes equations

Eduardo Casas, Luis A. Fernández \& Jiongmin Yong, Optimal control of quasilinear parabolic equations

Darrell Duffie, Jin Ma \& Jiongmin Yong, Black's consol rate conjecture

D.G. Aronson \& J.L. Vazquez, Anomalous exponents in nonlinear diffusion

Ruben D. Spies, Local existence and regularity of solutions for a mathematical model of thermomechanical phase transitions in shape memory materials with Landau-Ginzburg free energy

Pu Sun, On circular pipe Poiseuille flow instabilities

Angelo Favini, Mary Ann Horn, Irena Lasiecka \& Daniel Tataru, Global existence, uniqueness and regularity of solutions to a Von Kármán system with nonlinear boundary dissipation

A. Dontchev, Tz. Donchev \& I. Slavov, On the upper semicontinuity of the set of solutions of differential inclusions with a small parameter in the derivative

Jin Ma \& Jiongmin Yong, Regular-singular stochastic controls for higher dimensional diffusions - dynamic programming approach

1171 Alex Solomonoff, Bayes finite difference schemes

1172 Todd Arbogast \& Zhangxin Chen, On the implementation of mixed methods as nonconforming methods for second order elliptic problems

Zhangxin Chen \& Bernardo Cockburn, Convergence of a finite element method for the drift-diffusion semiconductor device equations: The multidimensional case

Boris Mordukhovich, Optimization and finite difference approximations of nonconvex differential inclusions with free time

Avner Friedman, David S. Ross, and Jianhua Zhang, A Stefan problem for reaction-diffusion system

Nikan B. Firoozye, Homogenization on lattices: Small parameter limits, $H$-measures, and discrete Wigner measures

G. Yin, Adaptive filtering with averaging

Wlodzimierz Byrc and Amir Dembo, Large deviations for quadratic functionals of Gaussian processes

Ilja Schmelzer, 3D anisotropic grid generation with intersection-based geometry interface

Alex Solomonoff, Application of multiple methods to two matrix eigenproblems

A.M. Latypov, Numerical solution of steady euler equations in streamline-aligned orthogonal coordinates

Bei Hu \& Hong-Ming Yin, Semilinear parabolic equations with prescribed energy

Bei Hu \& Jianhua Zhang, Global existence for a class of Non-Fickian polymer-penetrant systems

Rongze Zhao \& Thomas A. Posbergh, Robust stabilization of a uniformly rotating rigid body

Mary Ann Horn \& Irena Lasiecka, Uniform decay of weak solutions to a von Kármán plate with nonlinear boundary dissipation

Mary Ann Horn, Irena Lasiecka \& Daniel Tataru, Well-posedness and uniform decay rates for weak solutions to a von Kármán system with nonlinear dissipative boundary conditions

Mary Ann Horn, Nonlinear boundary stabilization of a von Kármán plate via bending moments only

Frank H. Shaw \& Charles J. Geyer, Constrained covariance component models

Tomasz Luczaka, A greedy algorithm estimating the height of random trees

Timo Seppäläinen, Maximum entropy principles for disordered spins

Yuandan Lin, Eduardo D. Sontag \& Yuan Wang, Recent results on Lyapunov-theoretic techniques for nonlinear stability

Svante Janson, Random regular graphs: Asymptotic distributions and contiguity

Rachid Ababou, Random porous media flow on large 3-D grids: Numerics, performance, \& application to homogenization 